OPEN ACCESS

Citation: Peterson P.M., Hasnaa A. Hosni, Eman K. Shamso (2020) A key to the grasses (Poaceae) of Egypt. Webbia. Journal of Plant Taxonomy and Geography 75(2): 329-353. doi: 10.36253/jopt-9004

Received: June 10, 2020

Accepted: August 8, 2020

Published: November 18, 2020

Copyright: ( 2020 Peterson P.M., Hasnaa A. Hosni, Eman K. Shamso. This is an open access, peer-reviewed article published by Firenze University Press (http://www.fupress.com/webbia) and distributed under the terms of the Creative Commons Attribution License, which permits unrestricted use, distribution, and reproduction in any medium, provided the original author and source are credited.

Data Availability Statement: All relevant data are within the paper and its Supporting Information files.

Competing Interests: The Author(s) declare(s) no conflict of interest.

Editor: Riccardo M. Baldini

\section{A key to the grasses (Poaceae) of Egypt}

\author{
Paul M. Peterson ${ }^{1, \star}$, Hasnaa A. Hosni ${ }^{2}$, Eman K. Shamso ${ }^{2}$ \\ ${ }^{1}$ Department of Botany MRC-166, National Museum of Natural History, Smithsonian \\ Institution, Washington DC 20013-7012, USA \\ ${ }^{2}$ Herbarium, Botany and Microbiology Department, Faculty of Science, Cairo University, \\ Giza 12613, Egypt \\ ${ }^{*}$ Corresponding author: peterson@si.edu
}

\begin{abstract}
A key for identifying 284 native and naturalized Egyptian grass species belonging to 103 genera in 22 tribes and 7 subfamilies is presented. The key is principally based on floral characters of the inflorescence and spikelet. A list and classification of all known species of Egyptian grasses is provided.
\end{abstract}

Keywords: classification, Gramineae, grasses, Egypt, identification key.

\section{INTRODUCTION}

The Poaceae (Gramineae) is a large cosmopolitan family with 768 genera and 11,506 species (Soreng et al. 2017). The family includes cereal grasses, bamboos, and species occurring in natural grasslands, cultivated lawns, and pastures. The family has been divided into subfamilies ranging from two (Tzvelev 1989) to six (Clayton and Renvoize 1986), and more recently the family has been divided into 12 subfamilies (Takhtajan 2009; Reveal 2012; Soreng et al. 2017, 2019; Stevens 2017).

In Egypt the grasses are the largest family of flowering plants with 284 species belonging to 103 genera and 22 tribes (Ibrahim et al. 2016). The most comprehensive account of the family in Egypt was done by Täckholm et al. (1941). Other treatments of the grasses of Egypt include Täckholm (1974), Cope and Hosni (1991), Cope (2005), Boulos (2009), and Ibrahim et al. (2016).

The identification of grasses is usually based on the structure of the inflorescence and floral characteristics. However, in some cases it is necessary to identify grasses by its vegetative character if the flowers are not available. In such cases the vegetative characters can be used until a flowering specimen is obtained (Hosni and Ibrahim 2004; Ibrahim et al. 2016).

Phylogenetic studies using results from DNA sequences have changed the classification of the grasses and this paper follows the current use of a name as proposed in these papers. With the publication of the grasses of Egypt using a vegetative key (Ibrahim et al. 2016), the need for an updated floral key is apparent. Earlier traditional treatments of the grasses of Egypt, 
i.e., Cope and Hosni (1991) and Cope (2005) are outdated and it is often difficult to determine the current use name. Our paper presents a new key for the identification of grasses native and adventive in Egypt and is written for use by both trained botanists and interested amateurs. Therefore, we have included an introduction defining many terms used in the key. The key is designed to facilitate the identification and is simplified as much as possible using characters based on the inflorescence and spikelet. Our key refers only to Egyptian specimens and, in addition, we include a updated classification of all grasses found in Egypt.

The accepted names follow the Catalogue of New World Grasses (Soreng et al. 2015, 2017) using terminology found in Kellogg (2015), Clayton et al. (2016), Ibrahim et al. $(2016,2018)$, and Herrera Arrieta and Peterson (2018). Because the inflorescence of grasses takes a variety of shapes, it is convenient to group them into categories based on their morphology. Accordingly, the identification key is divided into two parts, a key of major groups based mainly on inflorescence characters followed by keys to the species within each group. Brief descriptions, synonyms, and illustrations of the species was provided in Ibrahim et al. (2016). The classification of each species in Table 1 follows Soreng et al. (2017, 2019).

\section{GENERAL MORPHOLOGY}

Grasses are annual, biennial or perennial herbs and the root systems are fibrous, rhizomatous or stoloniferous. Flowering stems (culms) are usually unbranched, composed of several internodes and are mostly hollow, rarely solid throughout, and the solid nodes can sometimes be hairy. Leaves are borne solitary at the node and can be crowded at the base of the culm. Each leaf consists of a sheath, ligule and lamina. Leaf blades may be hairy or glabrous. The base of the leaf sheath is attached to the nodes and clasping the stems firmly with overlapping free or connate margins, sometimes with two small falcate or erect outgrowths at the mouth (auricles). At the junction of a sheath and blade is a ligule that can be membranous or hairy (often a line of hairs) but occasionally a ligule can be absent. Leaf sheaths are mostly linear, flat, and sometimes folded or rolled in various ways.

Flowers (Fig. 1) are usually hermaphrodite, sometimes unisexual (male and female), anemophilous (sometimes autogamous, apomictic or entomophilous) small and inconspicuous. The perianth is usually represented by two or three, minute but up to six, inconspicu- ous hyaline scales (lodicules) which correspond to the inner perianth whorl of other monocots. Stamens are hypogenous, 1-6 in number but usually 3 with delicate filaments and two anthers that dehisce through terminal pores or longitudinal slits. The ovary is unilocular with a single ovule. There are usually two or three (rarely 1) styles, generally with plumose stigmas. In grasses the fruit or caryopsis is single-seeded with an adherent pericarp, although there are numerous species with free pericarps and these would technically be termed akenes.

The floral parts are placed between two bracts, the lower (lemma) and upper (palea). These two structures are collectively referred to as a floret. The floret is usually subtended by two glumes. Lemmas vary in size and texture like the glumes and differ in the number of veins (usually with an odd number of veins), their overall shape, and the nature of their attachment to the rachilla. Lemmas are often awned or mucronate near the apex, or the awn is borne somewhere along the dorsal back. Awns can be straight, keeled or twisted. The paleas are usually membranous, tightly enclosing the pistil and stamens. Paleas (sometimes reduced) usually have two major veins and are therefore 2-keeled. The lemma, palea, and reproductive structures are called florets. The characteristic floral structure in grasses (spikelets) consist of one to many florets distichously inserted on either side of a slender, jointed rachilla (Fig. 1). Spikelets vary in size from minute (1 $\mathrm{mm}$ or less) to relatively large (1 or $2 \mathrm{~cm}$ ). Each spikelet is usually subtended by two lower empty scales or glumes. Glumes are variously veined and sometimes bear one or more awns. The base of a spikelet or floret is sometimes enlarged and hardened into a small knob or stalk (often sharp) called a callus. Glumes may be shorter than the adjoining lemma or longer and sometimes can be long enough to enclose the entire spikelet, or one or both glumes may be reduced or absent. Spikelets may be dorsiventrally compressed, laterally compressed, or terete.

Table 1. The following list is a synopsis of the classification of the genera into subfamilies and tribes for the grasses of Egypt.

\section{Subfamily: ARISTIDOIDEAE Caro}

1. TRIBE: ARISTIDEAE C.E.Hubb. Aristida adscensionis L. Aristida funiculata Trin. \& Rupr. Aristida mutabilis Trin. \& Rupr. Stipagrostis acutiflora (Trin. \& Rupr.) De Winter Stipagrostis ciliata (Desf.) De Winter Stipagrostis drarii (Täckh.) De Winter Stipagrostis hirtigluma (Steud. ex Trin. \& Rupr.) De Winter 
Stipagrostis lanata (Forssk.) De Winter

Stipagrostis multinerva $\mathrm{H}$. Scholz

Stipagrostis obtusa (Delile) Nees

Stipagrostis paradisea (Edgew.) De Winter

Stipagrostis plumosa (L.) Munro ex T. Anderson

Stipagrostis raddiana (Savi) De Winter

Stipagrostis scoparia (Trin. \& Rupr.) De Winter

Stipagrostis shawii (H.Scholz) H.Scholz

Stipagrostis uniplumis (Licht.) De Winter

Stipagrostis vulnerans (Trin. \& Rupr.) De Winter

II. Subfamily ARUNDINOIDEAE Kunth ex Beilschm.

2. TRIBE: ARUNDINEAE Dumort.

Arundo donax L.

3. TRIBE: MOLINIEAE Jirásek

Phragmites australis (Cav.) Trin. ex Steud.

Phragmites karka (Retz.) Steud. [syn Phragmites mauritianus Kunth]

III. Subfamily CHLORIDOIDEAE Kunth ex Beilschm.

4. TRIBE: CENTROPODIEAE P.M. Peterson, N.P. Barker \& H.P. Linder

Centropodia forskalii (Vahl) Cope

Centropodia fragilis (Guinet \& Sauvage) Cope

5. TRIBE: CYNODONTEAE Dumort.

Aeluropus lagopoides (L.) Trin. ex Thwaites

Aeluropus littoralis (Gouan) Parl.

Chloris flagellifera (Nees) P.M. Peterson [syn. Ochthoch-

loa compressa (Forssk.) Hilu]

Chloris gayana Kunth

Chloris prieurii Kunth [syn Enteropogon prieurii (Kunth)

Clayton]

Chloris pycnothrix Trin.

Chloris virgata Sw.

Coelachyrum brevifolium Hochst. \& Nees

Cynodon dactylon (L.) Pers.

Cynodon transvaalensis Burtt Davy

Dactyloctenium aegyptium (L.) Willd.

Dactyloctenium aristatum Link

Dactyloctenium scindicum Boiss.

Desmostachya bipinnata (L.) Stapf

Dinebra retroflexa (Vahl) Panz.

Dinebra panicea (Retz.) P.M.Peterson \& N.Snow [syn. Leptochloa panicea (Retz.) Ohwi]

Diplachne fusca (L.) P.Beauv. [syn. Leptochloa fusca (L.)

Kunth]

Eleusine africana Kenn.- O’Byrne
Eleusine coracana (L.) Gaertn.

Eleusine floccifolia (Forssk.) Spreng.

Eleusine indica (L.) Gaetn.

Halopyrum mucronatum (L.) Stapf

Leptothrium senegalense (Kunth) Clayton

Melanocenchris abyssinica (R.Br. ex Fresen.) Hochst.

Schoenefeldia gracilis Kunth

Tetrapogon cenchriformis (A.Rich.) Clayton

Tetrapogon villosus Desf.

Tragus berteronianus Schult.

Tragus racemosus (L.) All.

Trichoneura mollis (Kunth) E.Ekman

6. TRIBE ERAGROSTIDEAE Stapf

Enneapogon desvauxii P.Beauv.

Enneapogon lophotrichus Chiov. ex H.Scholz \& P.Koinig

Enneapogon persicus Boiss.

Enneapogon scaber Lehm.

Eragrostis aegyptiaca (Willd.) Delile

Eragrostis aspera (Jacq.) Nees

Eragrostis barrelieri Daveau

Eragrostis cilianensis (All.) Vign. ex Janch.

Eragrostis ciliaris (L.) R. Br.

Eragrostis japonica (Thunb.) Trin.

Eragrostis lepida (A.Rich.) Hochst. ex Steud.

Eragrostis minor Host

Eragrostis pilosa (L.) P.Beauv.

Eragrostis sarmentosa (Thunb.) Trin.

Eragrostis tef (Zucc.) Trotter

Eragrostis tenuifolia (A.Rich.) Hochst. ex Steud.

Eragrostis tremula Hochst. ex Steud.

Schmidtia pappophoroides Steud.

7. TRIBE TRIRAPHIDEAE P.M. Peterson

Triraphis pumilio R.Br.

8.TRIBE ZOYSIEAE Benth.

Sporobolus alopecuroides (Piller \& Mitterp.) P.M.Peterson [syn. Crypsis alopecuroides (Piller \& Mitterp.) Schrad.]

Sporobolus aculeatus (L.) P.M.Peterson [syn. Crypsis aculeata (L.) Aiton]

Sporobolus ioclados (Nees ex Trin.) Nees

Sporobolus natalensis (Steud.) T. Durand \& Schinz

Sporobolus niliacus (Bornm.) P.M.Peterson [syn. Crypsis

vaginiflora (Forssk.) Opiz]

Sporobolus spicatus ( Vahl) Kunth

Sporobolus pungens (Schreb.) Kunth

Sporobolus schoenoides (L.) P.M.Peterson [syn. Crypsis schoenoides (L.) Lam.]

Sporobolus wrightii Munro ex Scribn. 
IV. Subfamily DANTHONIOIDEAE H.P. Linder \& N.P. Barker

9. TRIBE: DANTHONIEAE Zotov.

Cortaderia selloana (Schult. \& Schult. f.) Asch. \& Graebn.

Schismus arabicus Nees

Schismus barbatus (L.) Thell.

V. Subfamily: ORYZOIDEAE Kunth ex Beilschm.

10. TRIBE: EHRHARTEAE Nevski

Ehrharta calycina Sm.

11. TRIBE: ORYZEAE Dumort.

Leersia hexandra Sw.

Oryza sativa $\mathrm{L}$.

\section{Subfamily PANICOIDEAE Link}

12. TRIBE: ANDROPOGONEAE Dumort.

Andropogon distachyos $\mathrm{L}$.

Chrysopogon plumulosus Hochst.

Chrysopogon zizanioides (L.) Roberty

Coix lacryma-jobi $\mathrm{L}$.

Cymbopogon citratus (DC.) Stapf

Cymbopogon flexuosus (Nees ex Steud.) Watson

Cymbopogon jwarancusa (Jones) Schult.

Cymbopogon martinii (Roxb.) Watson

Cymbopogon nardus (L.) Rendle

Cymbopogon schoenanthus (L.) Spreng. subsp. schoenanthus

Cymbopogon schoenanthus subsp. proximus

Dichanthium annulatum (Forssk.) Stapf

Dichanthium foveolatum (Delile) Roberty

Elionurus royleanus Nees ex A. Rich

Hemarthria altissima (Poir.) Stapf \& C.E.Hubb.

Hyparrhenia hirta (L.) Stapf

Imperata cylindrica (L.) Raeusch.

Lasiurus scindicus Henrard

Miscanthus sinensis Andersson

Pogonatherum paniceum (Lam.) Hack.

Saccharum officinarum L.

Saccharum spontaneum $\mathrm{L}$.

Sorghum arundinaceum (Desv.) Stapf

Sorghum bicolor (L.) Moench

Sorghum $x$ drummondii (Nees ex Steud.) Millsp. \& Chase

Sorghum halepense (L.) Pers.

Sorghum virgatum (Hack.) Stapf

Themeda triandra Forssk.

Themeda villosa (Poir.) A.Camus
Vossia cuspidata (Roxb.) Griff.

Zea mays L. subsp. mays

Zea mays subsp. mexicana (Schrad.) Iltis

13. TRIBE: PANICEAE R.Br.

Cenchrus americanus (L.) Morrone [syn. Pennisetum

glaucum (L.) R.Br.]

Cenchrus biflorus Roxb.

Cenchrus ciliaris L. [syn. Pennisetum ciliare (L.) Link]

Cenchrus clandestinus (Hochst. ex Chiov.) Morrone (syn.

Pennisetum cladestinum Hochst. ex Chiov.)

Cenchrus echinatus L.

Cenchrus longisetus M.C.Johnst. (syn. Pennisetum villo-

sum R. Br. ex Fresen.)

Cenchrus orientalis (Rich.) Morrone (syn. Pennisetum

orientale Rich.)

Cenchrus pennisetiformis Hochst. \& Steud.

Cenchrus ramosissimus Poir. (syn. Pennisetum ramosissimus Poir.)

Cenchrus setaceus (Forssk.) Morrone [syn. Pennisetum setaceum (Forssk.) Chiov.]

Cenchrus setiger Vahl

Cenchrus sieberianus (Schltdl.) Verloove [syn. Pennisetum sieberianum (Schltdl.) Verloove]

Cenchrus violaceus (Lam.) Morrone

Digitaria ciliaris (Retz.) Koeler

Digitaria nodosa Parl.

Digitaria sanguinalis (L.) Scop.

Digitaria velutina (Forssk.) P.Beauv.

Digitaria violascens Link

Echinochloa colona (L.) Link

Echinochloa crusgalli (L.) P.Beauv.

Echinochloa pyramidalis (Lam.) Hitchc. \& Chase

Echinochloa stagnina (Retz.) P.Beauv.

Megathyrsus maximus (Jacq.) B.K.Simon \& S.W.L.Jacobs

(syn. Panicum maximum Jacq.)

Melinis minutiflora P.Beauv.

Melinis repens (Willd.) Zizka

Moorochloa eruciformis (Sm.) Veldkamp [syn. Brachiaria

eruciformis (Sm.) Griseb.]

Panicum antidotale Retz.

Panicum coloratum L.

Panicum hygrochris Steud.

Panicum miliaceum L.

Panicum repens $\mathrm{L}$.

Panicum turgidum Forssk.

Setaria geminata [syn. Paspalidium geminatum (Forssk.)

Stapf]

Setaria italica (L.) P.Beauv.

Setaria megaphylla (Steud.) T.Durand \& Schinz

Setaria obtusifolia (Delile) Morrone [syn. Paspalidium obtusifolium (Delile) D.Simpson] 
Setaria pumila (Poir.) Roem. \& Schult.

Setaria verticillata (L.) P.Beauv.

Setaria viridis (L.) P. Beauv.

Stenotaphrum secundatum (Walter) Kuntze

Tricholaena teneriffae (L.f.) Link

Urochloa deflexa (Schumach.) H.Scholz [syn. Brachiaria

deflexa (Schumach.) C.E.Hubb. ex Robyns.]

Urochloa leersioides (Hochst.) H.Scholz \& Valdés [syn.

Brachiaria leersioides (Hochst.) Stapf]

Urochloa mutica (Forssk.) T.Q.Nguyen [syn. Brachiaria mutica (Forssk.) Stapf]

Urochloa panicoides P. Beauv.

Urochloa ramose (L.) T.Q.Nguyen [syn. Brachiaria ramosa (L.) Stapf]

Urochloa reptans (L.) Stapf [syn. Brachiaria reptans (L.) C.A. Gardner]

14. TRIBE: PASPALEAE J.Presl

Paspalum dilatatum Poiret

Paspalum distichum L.

Paspalum racemosum Lam.

15. TRIBE: TRISTACHYIDEAE Sánchez-Ken \& L.G. Clark

Danthoniopsis barbata (Nees) C.E.Hubb.

VII. Subfamily: POOIDEAE Benth.

16. TRIBE: BRACHYPODIEAE Harz

Brachypodium distachyon (L.) P.Beauv.

17. TRIBE: BROMEAE Dumort.

Bromus aegyptiacus Tausch

Bromus alopecuros Poir.

Bromus catharticus Vahl

Bromus danthoniae Trin. ex C.A.Mey.

Bromus diandrus Roth var. diandrus

Bromus diandrus var. rigidus (Roth) Sales

Bromus fasciculatus C.Presl

Bromus hordeaceus L.

Bromus inermis Leyss.

Bromus japonicus Thunb.

Bromus lanceolatus Roth

Bromus lepidus Holmb.

Bromus madritensis L.

Bromus pectinatus Thunb.

Bromus pulchellus Fig. \& De Not.

Bromus pumilio (Trin.) P.M. Sm. [syn Boissiera squarrosa

(Banks \& Sol.) Nevski]

Bromus rubens L.

Bromus scoparius L.
Bromus sterilis L.

Bromus tectorum L. subsp. tectorum

Bromus tectorum subsp. lucidus Sales

18. TRIBE: LYGEEAE J.Presl

Lygeum spartum Loefl. ex L.

19. TRIBE: MELICEAE Rchb.

Melica persica Kunth

20. TRIBE: POEAE R.Br.

Agrostis stolonifera L.

Alopecurus myosuroides Huds.

Ammochloa palaestina Boiss.

Avena barbata Pott ex Link subsp. barbata

Avena barbata subsp. wiestii (Steud.) Mansf.

Avena fatua L.

Avena longiglumis Durieu

Avena sativa $\mathrm{L}$.

Avena sterilis L.subsp. sterilis

Avena sterilis subsp. ludoviciana (Durieu) J.M.Gillet \& Magne

Briza maxima L.

Briza minor L.

Calamagrostis arenaria (L.) Roth [syn. Ammophila arenaria (L.) Link

Catapodium rigidum (L.) C.E.Hubb..

Corynephorus divaricatus (Pourr.) Breistr.

Cutandia dichotoma (Forssk.) Batt. \& Trab.

Cutandia maritima (L.) Barbey

Cutandia memphitica (Spreng.) Benth.

Cynosurus echinatus L.

Dactylis glomerata L.

Desmazeria philistaea subsp. rohlfsiana (Coss.) H.Scholz

Festuca brevis (Boiss. \& Kotschy) Asch., Schweinf. \&

Muschl. (syn. Vulpia brevis Boiss. \& Kotschy)

Festuca fasciculata Forssk. [syn. Vulpia fasciculata (Forssk.) Samp.]

Festuca bromoides L. [syn. Vulpia bromoides (L.) Gray]

Festuca myuros L. [syn. Vulpia myuros (L.) C.C.Gmel.]

Festuca pectinella Delile [syn. Vulpia pectinella (Delile)

Boiss.]

Gastridium phleoides (Nees \& Meyen) C.E.Hubb.

Holcus annuus Salzm.

Lagurus ovatus L.

Lamarckia aurea (L.) Moench

Lolium arundinaceum (Schreb.) Darbysh. (syn Festuca arundinacea Schreb.)

Lolium multiflorum Lam.

Lolium perenne L.

Lolium rigidum Gaudin

Lolium temulentum L. 


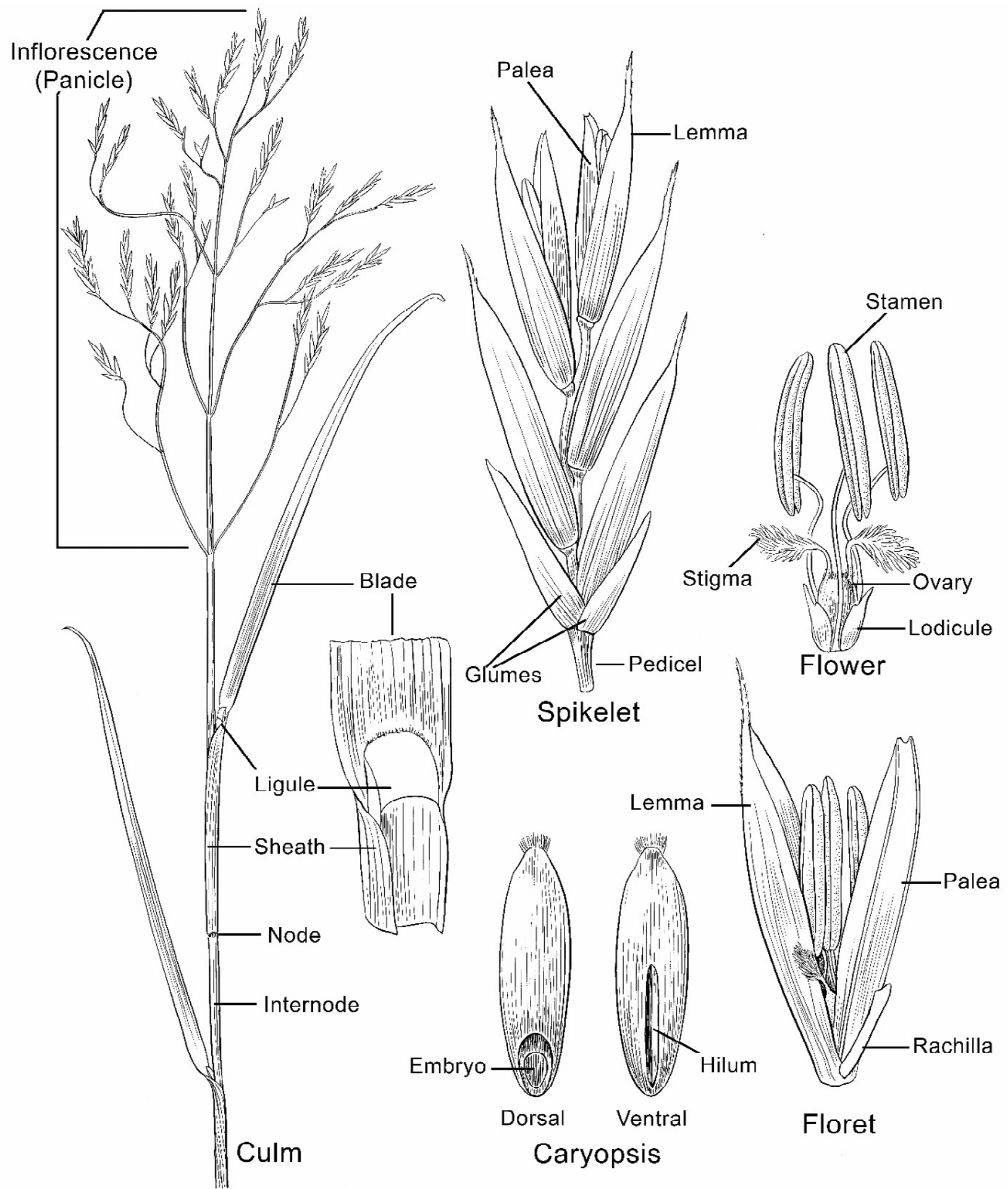

Figure 1. General morphology of a grass, illustrating the culm, blade, panicle, spikelet, floret, flower, and caryopsis.

Parapholis filiformis (Roth) C.E.Hubb.

Parapholis incurva (L.) C.E.Hubb.

Parapholis marginata Runem.
Phalaris aquatica $\mathrm{L}$.

Phalaris arundinacea $\mathrm{L}$.

Phalaris canariensis $\mathrm{L}$. 


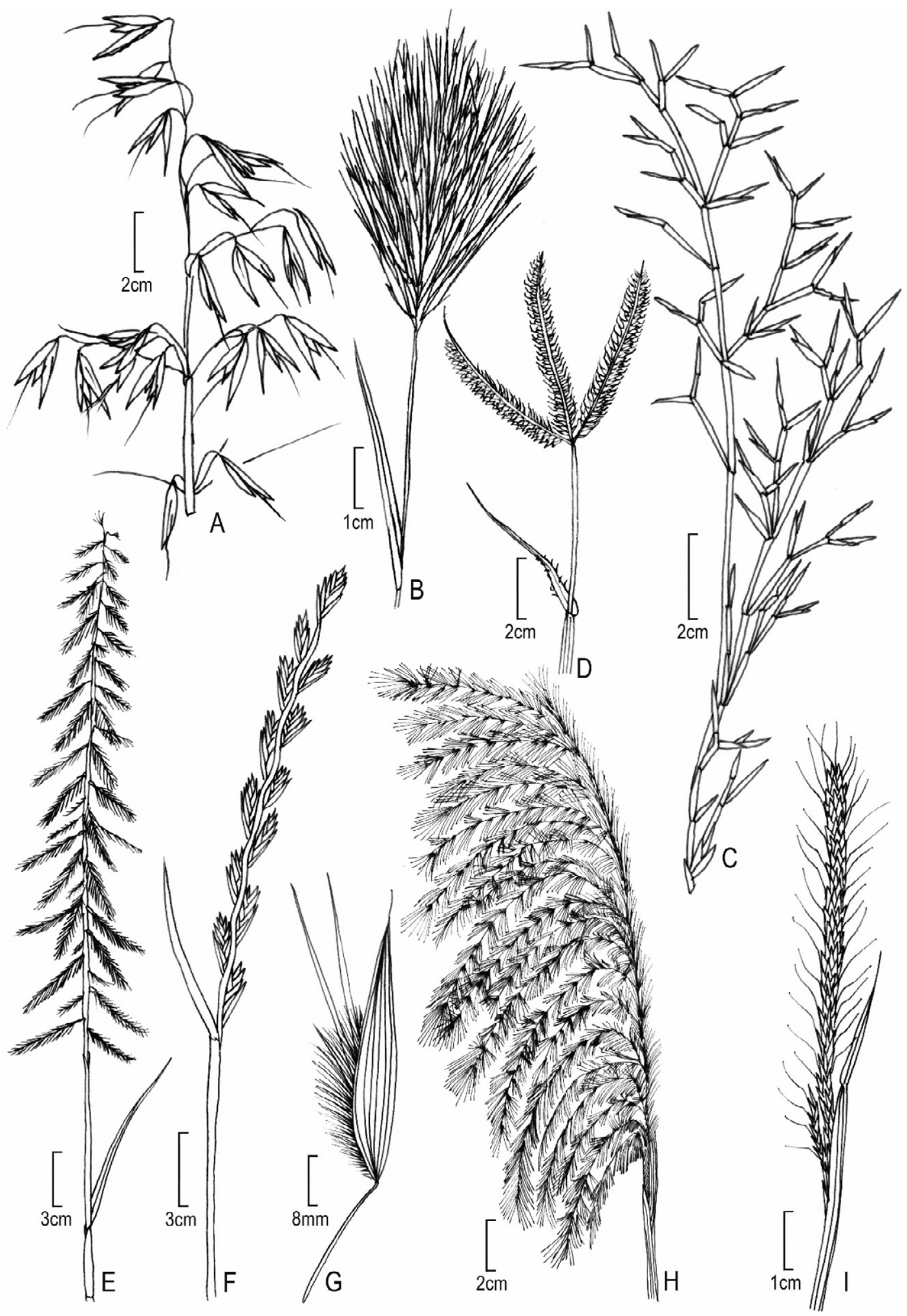

Figure 2. Inflorescence types used to determine groups in the key. A Open panicle (Avena sativa) B Headlike panicle (Bromus rubens) C Dichotomously branched panicle (Cutandia dichotoma) D Digitately arranged spikes (Dactyloctenium aegyptium) E Spikes along central axis (Dinebra retroflexa) F Simple spike (Lolium rigidum) G Panicles subtended by spatheole (Lygeum spartum) H Plumose panicle (Saccharum spontaneum) I Spiciform panicle (Trisetaria linearis). 
Phalaris coerulescens Desf.

Phalaris minor Retz.

Phalaris paradoxa $\mathrm{L}$.

Phleum pratense $\mathrm{L}$.

Phleum subulatum (Savi) Asch. \& Graebn.

Poa annua $\mathrm{L}$

Poa diaphora Trin. [syn. Eremopoa altaica (Trin.)

Roshev.]

Poa infirma Kunth

Poa persica Trin. [syn. Eremopoa persica (Trin.) Roshev.]

Poa sinaica Steudel

Polypogon maritimus Willd.

Polypogon monspeliensis (L.) Desf.

Polypogon viridis (Gouan) Breistr.

Rostraria cristata (L.) Tzvelev

Rostraria hispida (Savi) M.Dogan

Rostraria obtusiflora (Boiss.) Holub subsp. obtusiflora

Rostraria pumila (Desf.) Tzvelev

Rostraria rohlfsii (Asch.) Holub

Sphenopus divaricatus (Gouan) Rchb.

Triplachne nitens (Guss.) Link

Trisetaria glumacea (Boiss.) Maire

Trisetaria koelerioides (Bornm. \& Hack.) Melderis

Trisetaria linearis Forssk.

Trisetaria macrochaeta (Boiss.) Maire

21. TRIBE: STIPEAE Dumort.

Oloptum miliaceum (L.) Röser \& H.R.Hamasha [syn. Oryzopsis miliacea (L.) Asch. \& Schweinf.]

Piptatherum holciforme (M.Bieb.) Roem. \& Schult. [syn. Oryzopsis holciformis (M.Bieb.) Hack.]

Stipa arabica Trin \& Rupr.

Stipa lagascae Roem. \& Schult.

Stipellula capensis (Thunb.) Röser \& H.R.Hamasha (syn.

Stipa capensis Thunb.)

Stipellula parviflora (Desf.) Röser \& H.R.Hamasha (syn.

Stipa parviflora Desf.)

22. TRIBE: TRITICEAE Dumort.

Aegilops bicornis (Forssk.) Jaub. \& Spach

Aegilops geniculata Roth

Aegilops kotschyi Boiss.

Aegilops longissima Schweinf. \& Muschl.

Aegilops peregrina (Hack.) Eig

Aegilops ventricosa Tausch

Agropyron cristatum (L.) Gaertn.

Crithopsis delileana (Schult.) Roshev.

Elymus elongatus (Host) Runem.

Elymus farctus (Viv.) Runemark ex Melderis

Elymus repens (L.) Gould

Eremopyrum bonaepartis (Spreng.) Nevski

Eremopyrum distans (K.Koch) Nevski
Hordeum marinum Huds. subsp. marinum

Hordeum marinum subsp. gussoneanum

Hordeum murinum L. subsp. leporinum (Link) Arcang.

Hordeum murinum L. subsp. glaucum (Steud.) Tzvelev

Hordeum spontaneum $\mathrm{K}$. Koch

Hordeum vulgare $\mathrm{L}$.

Taeniatherum caput-medusae (L.) Nevski

Triticum aestivum $\mathrm{L}$.

Triticum dicoccum (Schrank) Schubl.

Triticum durum Desf.

Triticum pyramidale (Delile ex Schult.) Percival

Triticum turgidum L.

The inflorescence (synflorescence) is usually compound, composed of simple or complex aggregation of primary inflorescences (spikelets) often produced on a central axis (rachis) which may be terminal, rarely axillary, or compound and rebranched (Fig. 2). Spikelets may be arranged in spikes, racemes or panicles (open or contracted). In spikes, the spikelets are attached directly (sessile) to the unbranched main axis without pedicels. Racemes are unbranched inflorescences with each spikelet borne on a single pedicel directly on a branch axis. Multiple racemes can be arranged digitally or scattered along the rachis. Open or contracted panicles are inflorescences in which the main axis has several lateral, whorled or individual indeterminate branches with each branch terminating in a pedicellate spikelet. Spiciform panicles is where contraction has proceeded to the point where individual branches are closely appressed or adherent to the central axis. Inflorescences can sometimes be subtended by a bladeless sheath known as spatheole.

\section{IDENTIFICATION KEY}

The identification key is composed of two parts: a key to major groups and a key to each group.

Key to major groups

1a. Inflorescence ovoid, cylindrical or a headlike panicle ......... group 1

1b. Inflorescence open and not ovoid or a headlike panicle .. 2

2a. Inflorescence branches or spikes digitate or subdigitately inserted, terminal group 2

2b. Inflorescence branches branched and rebranched (paniculate) and not digitately or subdigitately inserted 3 
3a. Inflorescence with racemes or spikelike panicles terminal; spikelets with an involucre of numerous bristles or hairs at the base group 3

3b. Inflorescence with racemes appressed along a central axis or a panicle; spikelets without an involucre of numerous bristles or hairs at base...

... 4

4a. Spikes or racemes appressed along central axes .... group 4

4b. Spikes or racemes not appressed along central axes ........ 5

5a. Spikelets 3-9-awned or lemma awns 3-branched; inflorescence a contracted or open panicle group 5

5b. Spikelets 1-awned or unawned; inflorescence a raceme or panicle

6a. Spikelets prickly; inflorescence a simple raceme or spikelike panicle group 6

6b. Spikelets not prickly; inflorescence a raceme or panicle .. 7

7a. Inflorescence tri- or dichotomously branched ....... group 7

7b. Inflorescence not tri- or dichotomously branched ............. 8

8a. Inflorescence subtended by spatheoles. group 8

8 b. Inflorescence not subtended by spatheoles. 9

9a. Inflorescence plumose, large 20-60 cm long, open; culms 4-8 $\mathrm{m}$ tall group 9

9b. Inflorescence not plumose, generally $\leq 20(-60 \mathrm{~cm})$ long, if longer, never plumose, open or contracted; culms $\leq 2 \mathrm{~m}$ tall

10a. Inflorescence a simple spike, raceme or spikelike panicle... group 10

10b. Inflorescence an open or contracted panicle. 11

11a. Panicles spiciform, narrow usually $<1 \mathrm{~cm}$ wide group 11

11b. Panicles open usually $0.5-30 \mathrm{~cm}$ wide. 12

12a. Panicles contracted, $0.5-3(-4) \mathrm{cm}$ wide group 12

12b. Panicles open, not contracted, usually (2-) 4-30 cm wide.

13a. Spikelets awned group 13

13b. Spikelets unawned. group 14

Group 1: Inflorescence ovoid, cylindrical or a headlike panicle

1a. Heads woolly with plumose hairs or bristles .2

1b. Heads not wooly . .3 2a. Spikelets not subtended by bristles, 1-flowered with a single perfect floret; glumes (5.5-) 7-10 mm long, awned, the awns $1.5-3 \mathrm{~mm}$ long. Lagurus ovatus

2b. Spikelets subtended by an involucre of bristles, 2-flowered, the lower floret sterile; glumes $0.3-5.2 \mathrm{~mm}$ long, unawned .Cenchrus longisetus

3a. Spikelets subtended by an involucre of bristles, bristles plumose, $4-7 \mathrm{~cm}$ long ..Cenchrus longisetus

3b. Spikelets not subtended by an involucre of bristles ......... 4

4a. Panicle bilateral, pyramidal; cultivated Triticum pyramidale

4b. Panicle not pyramidal; native .5

5a. Stoloniferous perennials; with stringlike culms, richly branched at the nodes Aeluropus lagopoides

5b. Plants not stoloniferous; culms erect....... 6

6a. Glumes shiny with attenuate apices; lemmas shiny, surface villous, hairy below, hairs $4-8 \mathrm{~mm}$ long, apex setaceous or attenuate. Cortaderia selloana

6b. Glumes and lemmas green and not shiny, with acute and/ or mucronate apices... . .7

7a. Panicles subtended by an inflated sheath,or spatheole; spikelets 1-flowered

7b. Panicles not subtended by an inflated leaf sheath or spatheole; spikelets 2-many-flowered.

8a. Panicles ellipsoid; lower glume with glabrous margins ....... Sporobolus schoenoides

8b. Panicles ovoid or ellipsoid, lower glumes with hairy margins.

9a. Panicles 0.5-1 cm long; uppermost leaf continuous with its sheath; paleas $0-1$-veined Sporobolus aculeatus

9b. Panicles $0.3-1.5 \mathrm{~cm}$ long; uppermost leaf clearly demarcated from its sheath; paleas 2-veined ..Sporobolus niliacus

10a. Lemmas unawned 11

10b. Lemmas awned. 12

11a. Spikelets 4-14-flowered; panicles exserted Ammochloa palaestina

11b. Spikelets 3-flowered with only one fertile floret; panicles partially included in the sheath Phalaris minor

12a. Glumes dissimilar; lemmas mucronate or short-awned, the awns $\leq 1.5 \mathrm{~mm}$ long Dactylis glomerata

12b. Glumes similar; lemmas all awned, awns $\geq 2 \mathrm{~mm}$ long. 13 
13a. Most florets perfect; sheaths pubescent to pilose ........... 14

13b. Florets both sterile and perfect; sheaths glabrous .15

14a. Spikelets $15-30 \mathrm{~mm}$ long; lemmas 10-15 mm long, awns 7-23 mm long Bromus rubens

14b. Spikelets 4-5 mm long; lemmas 3-4. 5 mm long, awns 3-5 $\mathrm{mm}$ long Rostraria hispida

15a. Awns of sterile spikelets $15-20 \mathrm{~mm}$ long; spikelets 6-7 $\mathrm{mm}$ long; lemmas 3.4-4 mm long....... Cynosurus coloratus

15b. Awns of sterile spikelets 6-15 mm long; spikelets 8-14 $\mathrm{mm}$ long; lemmas 4-6.5 mm long...... Cynosurus echinatus

Group 2: Inflorescence branches or spikes digitate or subdigitately inserted, terminal

1a. Racemes 1-2 (3) digitate .................................................. 2

1b. Racemes usually 2 or more................................................. 8

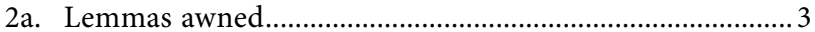

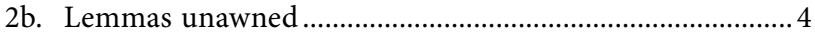

3a. Racemes subtended by reddish spatheole; rachis with white hairs; lemma awns 10-35 mm long, geniculate with twisted column Hyparrhenia hirta

3b. Racemes not subtended by spatheole; rachis glabrous; lemma awns 7-10 $\mathrm{mm}$ long, straight.

Brachypodium distachyon

4a. Culms spongy; racemes 10-30 cm long; rachis angular; glumes with long caudate apex Vossia cuspidata

4b. Culms not spongy; racemes $\leq 15.5 \mathrm{~cm}$ long; rachis winged or flattened; glumes without a caudate apex.......... 5

5a. Spikelets 4-7-flowered, 3.3-7.8 mm long.......................... 6

5b. Spikelets 1 or 2-flowered, when 2-flowered the lower floret sterile, $2-3.2 \mathrm{~mm}$ long.

6a. Leaf blades with tufts of short white hairs scattered along the margins; upper glumes 1veined; grain rugulose Eleusine floccifolia

6b. Leaf blades without tufts of hairs scattered along the margins; upper glumes 2-3-veined; grains striate....

Eleusine indica

7a. Racemes 2-8 cm long; rachis narrowly winged; spikelets dorsally compressed. .Paspalum distichum

7b. Racemes 0.7-1.5 cm long; rachis flattened; spikelets laterally compressed Cynodon transvaalensis

8a. Lemmas awned. . .9 8b. Lemmas unawned or short aristate ................................. 16

9a. Lemmas with geniculate awns and twisted columns....... 10

9b. Lemmas with straight or flexuous awns.

10a. Upper glume awned, the awn 4-10 mm long; fertile spikelets $8-16 \mathrm{~mm}$ long Andropogon distachyos

10b. Upper glume unawned; fertile spikelets $2-7 \mathrm{~mm}$ long... 11

11a. Racemes 3-7 cm long; lemma apex entire, awns 8-25 mm long, adventive Dichanthium annulatum

11b. Racemes 10-30 cm long; lemmas apex bifid, awns 6-12 $\mathrm{mm}$ long, cultivated. Miscanthus sinensis

12a. Lemmatal awns flexuous,10-30 (-40) mm long; glumes 1-awned, awns 0.5-1 mm long ......... Schoenefeldia gracilis

12b. Lemmatal awns straight, less than $10 \mathrm{~mm}$ long..... 13

13a. Lemmatal awns $0.5-5 \mathrm{~mm}$ long, as long as lemma Chloris gayana

13b. Lemmatal awns more than $5 \mathrm{~mm}$ long, more than twice as long as lemma .14

14a. Leaf blade with an obtuse apex; glume apex acute Chloris pycnothrix

14b. Leaf blade with an acute apex; glume apex acuminate ... 15

15a. Lowest lemma without a crown of spreading hairs; spikelets 4-6-flowered. Chloris prieurii

15b. Lowest lemma with a crown of long spreading hairs, the hairs 1.5-4 mm long; spikelets 3 flowered ..Chloris virgata

16a. Flowers unisexual, plants monoecious, female inflorescences subtended by leafy spatheoles, rachis enlarged into a woody cob

16b. Flowers perfect, plants hermaphroditic, inflorescences not subtended by spatheoles and rachis not enlarged into a cob

17a. Female inflorescences $2-5(-10) \mathrm{cm}$ thick with $8-24$ rows of spikelet pairs forming a thick woody cob, all tightly enclosed by several leafy spatheoles

Zea mays subsp. mays

17b. Female inflorescences less than $1 \mathrm{~cm}$ thick with 2 rows of spikelet pairs formang a hardened rachis, all enclosed by a single leafy spatheole. Zea mays subsp. mexicana

18a. Racemes 1-2 mm broad 19

18b. Racemes $2-5 \mathrm{~mm}$ broad 23

19a. Glumes equal; racemes 3-5. Chloris flagellifera

19b. Glumes unequal or absent; racemes 2-16. 20 
20a. Spikelets 1-flowered; racemes 2 or more closely placed or whorled with a flat rachis, unwinged; ligule a ciliate membrane Cynodon dactylon

20b. Spikelets 2-flowered; racemes mostly in pairs or whorled or alternate, he rachis flat or triquetrous, winged; ligule membranous ...21

21a. Glumes two, dissimilar Digitaria ciliaris

21b. Glume one, the lower absent or obscure .22

22a. Spikelets 2.5-3.3 mm long; fertile spikelets paired along a narrowly winged rachis. Digitaria sanguinalis

22b. Spikelets 1.2-2 mm long; fertile spikelets ternate along a broadly winged rachis Digitaria violascens

23a. Racemes terminated by the naked tips of the rachis, bristle like.

23b. Racemes terminated by a spikelet. .26

24a. Racemes 1.2-6.5 cm long, inflorescence open Dactyloctenium aegyptium

24b. Racemes $0.8-2 \mathrm{~cm}$ long, inflorescence compact...... .25

25a. Stoloniferous perennials; anthers 1.1-2 mm long Dactyloctenium scindicum

25b. Tufted annuals often rooting at the lower nodes; anthers $0.3-0.5 \mathrm{~mm}$ long.

Dactyloctenium aristatum

26a. Racemes in terminal pairs (rarely 3) with scattered one or two below; spikelets 8-20-flowered ......Acrachne racemosa

26b. Racemes not in terminal pairs, digitate or subdigitately arranged; spikelets 3-9-flowered 27

27a. Upper glume apex obtuse; lemma apex obtuse . Coelachyrum brevifolium

27b. Upper glume apex acute; lemma apex acute .28

28a. Racemes 9-15 mm wide; spikelets ovate; grains subglobose, brown, exposed between gaping lemmas and paleas at maturity, cultivated Eleusine coracana

28b. Racemes 3-8 mm wide; spikelets elliptic; grains ellipsoid, blackish, not exposed or concealed by the florets, native and/or weedy..... 29

29a. Leaf blades with tufts of short white hairs scattered along the margins; upper glumes 1veined; grain rugulose .... Eleusine floccifolia

29b. Leaf blades without tufts of hairs scattered along the margins; upper glumes 2-3-veined; grains striate or granular ...

30a. Lemmas 2.1-3.6 $\mathrm{mm}$ long; racemes 3-3.5 $\mathrm{mm}$ wide; grains $1-1.3 \mathrm{~mm}$ long, striate Eleusine indica 30b. Lemmas 3.7-5 mm long; racemes $4-8 \mathrm{~mm}$ wide; grains 1.2-1.6 mm long, granular Eleusine africana

Group 3: Inflorescence with racemes or spikelike panicles terminal; spikelet with an involucre of numerous bristles or hairs at the base

1a. Inflorescence 1 or 2 digitate racemes; spikelets surrounded by hairs; lemmatal awns 2-4 mm long, straight....

Tetrapogon villosus

1b. Inflorescence a panicle; spikelets surrounded by free or connate bristles; lemmas unawned

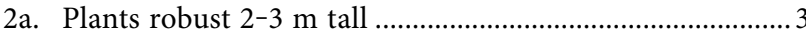

2b. Plants $90 \mathrm{~cm}$ or less tall .......................................................

3a. Annuals; culms glabrous below the panicle; bristles shorter than the spikelets, persistent; cultivated

.Cenchrus americanus

3b. Perennials; culms villous below the panicle; bristles much longer than the spikelets, deciduous; indigenous

Cenchrus orientalis

4a. Bristles connate, deciduous with the spikelets, inner bristles longer than outer with one long bristle, flat 5

4b. Bristles free, not deciduous with the spikelet...................... 9

5a. Bristles of the involucre retrorsely barbellate, tenaciously clinging to clothing, longest bristles scarcely emergent ...6

5b. Bristles of the involucre antrorsely scaberulous not clinging; sometimes with one conspicuous longer bristle ....... 7

6a. Inner bristles connate only at the bases to form a shallow disc, 2-4 mm long; inner bristle 2.9-7 mm long; ciliate ....

Cenchrus biflorus

6b. Inner bristles fused for about half their length to form a cup, 5-10 mm long; longest bristle 2-5 mm long, pubescent spinose . Cenchrus echinatus

7a. Inner bristles rigid, flattened, $2-3 \mathrm{~mm}$ long, connate for $1 / 2-2 / 3$ their length forming a cup, inner bristles with longest bristle scarcely emergent; $2-4 \mathrm{~mm}$ long.

....Cenchrus setiger

7b. Inner bristles flexuous, filiform above, 6-12 $\mathrm{mm}$ long, inner bristles with one conspicuously longer bristle 8-16 $\mathrm{mm}$ long; ciliate

8a. Inner bristles united only at the bases to form shallow disc $0.5-1.5 \mathrm{~mm}$ diam., occasionally connate for up to 0.5 $\mathrm{mm}$ above its rim Cenchrus ciliaris

8b. Inner bristles connate for 1-2.5 $\mathrm{mm}$ above the rim of the basal disc forming a cup Cenchrus pennisetiformis 
9a. Bristles persistent 10

9b. Bristles deciduous with the spikelets. 14

10a. Inflorescences open panicles, bristles $2-2.5 \mathrm{~cm}$ long Setaria megaphylla

10b. Inflorescences a spiciform panicles .11

11a. Bristles retrosely barbed clinging to clothes, 4-7 mm long Setaria verticillata

11b. Bristles antrorsely barbed 12

12a. Upper glumes as long as the spikelets; each spikelet subtended by 1-3 bristles, $5-10$ times as long as the spikelets. Setaria viridis

12b. Upper glumes shorter than the spikelets .13

13a. Lemmas strongly rugose; each spikelets subtended by 4-12 bristles, 3-8 $\mathrm{mm}$ long Setaria pumila

13b. Lemmas rugulose or unwrinkled; each spikelet subtended by 1-3 bristles, $4-16 \mathrm{~mm}$ long..... Setaria italica

14a. Inflorescences comprising only a few spikelets; comprising 2-4(-6) fertile spikelets; shorter than basal leaves; subtended by an inflated leaf-sheaths; enclosed; bristles shorter than the spikelets (cult.) ....Cenchrus clandestinum

14b. Inflorescences spike-like panicles, exserted. 15

15a. Panicles ovoid to subspherical very dense;bristles plumose, 40-70 mm long; lemmas acute, 7-9-veined

...Cenchrus longisetus

15b. Panicles elongate; lemmas .16

16a. Involucre sessile Cenchrus violaceum

16b. Involucre stipitate (stalked. 17

17a. Plants glaucous, pinkish; spikes purple or pink; involucral base stipitate, the stipe 1-3 mm long.... Cenchrus setaceus

17b. Plants not glaucous, not pinkish; involucral base stipitate, the stipe $0.5-1 \mathrm{~mm}$ long..... 18

18a. Panicles axes glabrous; involucral bristles 7-20 mm long .. ..Cenchrus ramosissimus

18b. Panicles axes pubescent; involucral bristles $6-9 \mathrm{~mm}$ long with a conspicuous longer bristle, $10-25 \mathrm{~mm}$ long

Cenchrus sieberianus

\section{Group 4: Spikes or racemes appressed along central axes}

1a. Spikelets headlike, several, small, nodding along an unbranched axes; glumes long villous and soft Melanocenchris abyssinica 1b. Spikelets not headlike, erect or reflexed along the central axis but not nodding; glumes not long villous

2a. Glumes as long as or longer than the spikelets, persistent, similar, exceeding apex of florets; lower glume apex caudate

Dinebra retroflexa

2b. Glumes much shorter than the spikelets; lower glume apex not caudate. 3

3a. Racemes $0.5-1 \mathrm{~cm}$ long; spikelets embedded in a corky rachis; leaf blade apex obtuse....Stenotaphrum secundatum

3b. Racemes more than $1 \mathrm{~cm}$ long, not embedded in rachis; leaf blades apex acute.

4a. Lemmas and/or glumes acuminate or awned .................... 5

4b. Lemmas or glumes unawned

5a. Racemes 15-35 cm long; spikelets 8-15 mm long; lemma apex mucronate or with a short awn $\leq 1.5 \mathrm{~mm}$ long; fertile spikelets pedicelled, pedicels filiform, 0.5-1.5 mm long; glumes unequal. Diplachne fusca

5b. Racemes 2-10 cm long; spikelets 3-6 mm long; lemma apex awned; fertile spikelets sessile or with pedicels $<0.5$ mm long; glumes equal or subequel.

6a. Lemmas apex mucronate or short awned, the awns 0.3-3 $\mathrm{mm}$ long.

6b. Lemmas awned, the awn 20-50 mm long 8

7a. Racemes 2-7(-10); spikelets comprising 1 fertile floret; spikelets 3-4.5 mm long; lemmas rugulose, mucronate, the mucro $0.3-1 \mathrm{~mm}$ long Urochloa panicoides

7b. Racemes 10-40; spikelets comprising 5-9 fertile florets; spikelets 6-8 mm long; lemmas sparingly hairy, mucronate or short awned up to $2 \mathrm{~mm}$ long ...Trichoneura mollis

8a. Caespitose annuals; racemes straight; spikelets pedicelled, 1-2 $\mathrm{mm}$ long; lemmas hispid, awns up to $50 \mathrm{~mm}$ long; upper glumes apex cuspidate Echinochloa crusgalli

8b. Rhizomatous perennials; racemes flexuous; spikelets sessile to subsessile; lemmas pubescent, awns $20 \mathrm{~mm}$ long, upper glumes entire or awned, the awns 0-4 $\mathrm{mm}$ long......

Echinochloa stagnina

9a. Rachis winged 10

9b. Rachis unwinged 15

10a. Racemes compact, appressed to the long slightly hollowed common axis; leaf blade apex obtuse ..... Setaria obtusifolia

10b. Racemes not compact, and not as above; leaf apex acute11

11a. Spikelets 6-9 mm long; lemma margin and midvein pubescent; glume apex caudate Dinebra panicea 
11b. Spikelets 1.5-4 mm long; lemmas glabrous; glume apex acute to obtuse

12a. Spikelets 2.5-3.5 mm long; rachis broadly winged, the wing $0.5-2 \mathrm{~mm}$ wide; lemmas apex obtuse

12b. Spikelets $1.5-2.5 \mathrm{~mm}$ long; rachis narrowly winged, the wing $<0.5 \mathrm{~mm}$ wide; lemma apex acute .15

13a. Lower glumes absent or obscure....... Paspalum racemosum

13b. Both glumes present, dissimilar .14

14a. Racemes 5-20 cm long; spikelets 2.5-3.5 mm long; lemma surfaces rugulose, stramineous Urochloa mutica

14b. Racemes 0.5-4 cm long; spikelets $1.5-2.5 \mathrm{~mm}$ long; lemma surface smooth, shiny, dark brown.

Setaria geminata

15a. Glume one, the lower absent or obscure 16

15b. Glumes 2, the lower present .17

16a. Glumes apex obtuse; lemmas 3-veined Paspalum dilatatum

16b. Glumes apex acute; lemmas 7-veined ..... Digitaria velutina

17a. Inflorescences up to $60 \mathrm{~cm}$ long, racemes numerous 20-50 or more Desmostachya bipinnata

17b. Inflorescences $<60 \mathrm{~cm}$ long, racemes few, up to $10 \ldots \ldots . . .18$

18a. Lemma and glume apices obtuse... Moorochloa eruciformis

18b. Lemma and glume apices acute or cuspidate .19

19a. Spikelets with 8-25 florets. .20

19b. Spikelets with 1 fertile and 1 sterile floret 21

20a. Rachilla villous; callus bearded.... Halopyrum mucronatum

20b. Rachilla glabrous; callus not bearded Catapodium rigidum

21a. Lower glume apex obtuse; lemmas pilose or villous, bearing white hairs

..Digitaria nodosa

21b. Lower glume apex acute; lemmas not pilose or villous ..22

22a. Spikelets packing adaxial, distant, irregular.

Urochloa deflexa

22b. Spikelets packing regular, 2- or 4-6-rowed .23

23a. Spikelets packing regular, 2-rowed .24

23b. Spikelets packing regular, 4-6-rowed .27

24a. Spikelets packing broadside to rachis; glume apex obtuse and erose, lemma apex apiculate Aeluropus littoralis 24b. Spikelets not packing broadside to rachis; glume apex acute, not erose; lemmas apex acute, often mucronate .....25

25a. Spikelets $1.5-2 \mathrm{~mm}$ long with hirsute pedicels; lower glumes $0.15-0.25$ as long as the spikelets without veins, apex truncate

Urochloa reptans

25b. Spikelets $2-3.5 \mathrm{~mm}$ long with glabrous or scabrous pedicels; lower glumes $1 / 3-1 / 2$ as long as the spikelets with $3-7$ veins, apex acute or obtuse

26a. Lemmas dark brown; upper glumes not separated from lower glumes by an internode Urochloa ramosa

26b. Lemmas green; the upper glumes separated from the lower glumes by a distinct internode $0.2-0.5 \mathrm{~mm}$ long...... ..Urochloa leersioides

27a. Plants annual, caespitose; upper glumes pubescent, apex cuspidate Echinochloa colona

27b. Plants perennial with rhizomes; upper glumes glabrous or hispidulous, apex acute Echinochloa pyramidalis

Group 5: Spikelets 3-9-awned or lemma awns 3-branched; inflorescence a contracted or open panicle

1a. Lemmas 5-9-awned .2

1b. Lemmas awns 3, or awns 3-branched 7

2a. Panicles open or contracted; lemmas hairy below the middle, 5-awned, the awns 4-8 mm long..... Schmidtia pappophoroides

2b. Panicles compact or spiciform; lemmas not hairy; lemmas 5-9-awned 3

3a. Small villous grasses; panicles densely ovoid; lemmas with 5-9 scabrid awns Bromus pumilio

3b. Tall, glabrous grasses, panicles spiciform, oblong-linear; lemma awns 9 , feathery.... .. 4

4a. Lemmas awns scaberulous throughout Enneapogon scaber

4b. Lemmas awns ciliate below, scaberulous only towards tip.. 5

5a. Fertile lemmas with 3 dense patches of hairs on the backs, one along midrib and one along each margin; nodes glabrous Enneapogon lophotrichus

5b. Lemmas with hairs on the back evenly distributed; nodes bearded 6

6a. Basal leaf sheaths persistent, forming a bulbous swelling near base; spikelets $2.8-5.5 \mathrm{~mm}$ long Enneapogon desvauxii 
7a. Basal leaf sheaths persistent, not forming a bulbous swelling near base; spikelets $5.5-12 \mathrm{~mm}$ long. Enneapogon persicus

7a. Lemma awns glabrous to scabrous, column not twisted .. 8

7b. Lemma awns feathery, at least the principal or central awn with atwisted column... ... 14

8a. Lemma 3-awned, central awn arising from sinus and the lateral awns arising dorsally or marginally, persistent, central awn not more than $10 \mathrm{~mm}$ long, lateral awns not more than $5 \mathrm{~mm}$ long

8b. Lemma with a single 3-branched awn, deciduous, central awn more than $10 \mathrm{~mm}$ long, lateral awns usually more than $5 \mathrm{~mm}$ long. .. 15

9a. Central lemma awns geniculate with twisted column, apex of awn smooth. 10

9b. Central lemma awn straight, apex of awn scabrous ........ 13

10a. Central lemma awns inserted below the middle, scarcely exserted from the spikelet, the awns 2.5-3 mm long, lateral lemma awns 1-1.5 mm long....... Trisetaria glumacea

10b. Central lemma awns inserted near the middle or upper $1 / 4$, exserted from the spikelet, the awns 3-10 mm long, lateral lemma awns 1-5 mm long. .. 11

11a. Lemmas glabrous, central awns 5-7 mm long; lateral awns 1.5-5 mm long

11b. Lemmas villous, central awns $3-4 \mathrm{~mm}$ long, lateral awns 1-1.5 mm long. Triplachne nitens

12a. Panicles loosely contracted, $0.5-3.5 \mathrm{~cm}$ wide, oblongovate; central lemma awn 5-8 mm long, lateral awns 1.5 $\mathrm{mm}$ long Trisetaria macrochaeta

12b. Panicles densely contracted, $0.5-1 \mathrm{~cm}$ wide, linear; central lemma awn 8-15 $\mathrm{mm}$ long, lateral awns 3-5 mm long ....... Trisetaria linearis

13a. Spikelets elliptic, $10-40 \mathrm{~mm}$ long; lemmas with minutely bifid apex, awns curved, spreading, 5-15 mm long, lateral lemma awns arising dorsally, shorter than the central

Bromus danthoniae

13b. Spikelets lanceolate, 4-6 mm long; lemmas central awns 1.5-3 mm long, lateral lemma awns arising from the margins $0.5-1 \mathrm{~mm}$ long

14a. Spikelets 5-11-flowered; glumes 1.5-3 mm long, shorter than the spikelet; lemmas pilose, margins ciliate, central awn inserted in the sinus, the awn 1.5-2.5 mm long, not geniculate or twisted Triraphis pumilio

14b. Spikelets 2-flowered; glumes 4-5 mm long, longer than the spikelet; lemmas entirely glabrous, shiny, central awn inserted dorsally on upper $1 / 2$, the awn $2.5-3 \mathrm{~mm}$ long, geniculate and twisted

Trisetaria glumacea 15a. All three awns glabrous, lateral awns as long as the central awn; glumes unequal...................................................... 16

15b. Central and sometimes lateral awns plumose, lateral awns shorter than the central; glumes subequal 18

16a. Awns without a column, not articulating at the summit; ligule a ciliolate membrane. Aristida adscensionis

16b. Awns with a column, articulating at the summit; ligule a fringe of hairs 17

17a. Spikelets 6-7 mm long; lemma awns 10-30 mm mm long ..Aristida mutabilis

17b. Spikelets $20-30 \mathrm{~mm}$ long; lemma awns 35-45 $\mathrm{mm}$ long .... Aristida funiculata

18a. All three lemma awns plumose, lateral awns about as long as the central awn with or without a twisted column .... 19

18b. Only the central lemma awn plumose, lateral awns much shorter than the central awn with a twisted column ......21

19a. Internodes densely woolly; panicles $5-10 \mathrm{~cm}$ long; central lemma awns 20-35 mm long, the column 3-5 mm long, twisted Stipagrostis lanata

19b. Internodes glabrous; panicles 10-40 cm long; central lemmas awns 7-10 $\mathrm{mm}$ long, the column 0-1 $\mathrm{mm}$ long, straight or slightly twisted .20

20a. Lower glumes longer than the upper; panicles $20-40 \mathrm{~cm}$ long; awns not or scarcely exserted from spikelets.

..Stipagrostis scoparia

20b. Lower glumes shorter than the upper; panicles $10-20 \mathrm{~cm}$ long; awns exserted from the spikelets.

Stipagrostis vulnerans

21a. Nodes bearded; lemmas articulating near the middle (across the body) Stipagrostis ciliata

21b. Nodes not bearded; lemmas articulating near apex. 22

22a. Central lemma awns plumose throughout..... 23

22b. Central lemma awns glabrous in the lower and/or upper portions

23a. Callus glabrous; column straight or slightly twisted Stipagrostis raddiana

23b. Callus bearded; column twisted. 24

24a. Callus with 2 collars of hairs; column 7-13 mm long, hairy on the upper portion; central lemma awns 35-70 $\mathrm{mm}$ long, lateral lemma awns 10-25 mm long. Stipagrostis hirtigluma

24b. Callus with a single collar of hairs; column 5-10 mm long, glabrous; central lemma awns 20-35 mm long, lateral lemma awns 10-12 mm long ....Stipagrostis uniplumis 
25a. Central lemma awns glabrous in the lower or upper portion.

25b. Central lemma awns glabrous in the lower and upper portion

26a. Central lemma glabrescent toward tip

26b. Central lemma awns glabrescent toward base

27a. Callus glabrous; column 1.5-2 mm long; central lemma awns 13-25 mm long, lateral lemma awns 7-17 mm long Stipagrostis drarii

27b. Callus bearded; column 11-14 mm long; central lemma awns 60-70 mm long, lateral lemma awns 17-25 mm long Stipagrostis paradisea

28a. Lower glume apex obtuse; lemmas 2-2.5 mm long, central lemma awns 20-30 mm long, Stipagrostis obtusa

28b. Lower glume apex acute; lemmas 3-4 mm long, central lemma awns 45-47 $\mathrm{mm}$ long Stipagrostis shawii

29a. Column straight or slightly twisted, $1 \mathrm{~mm}$ long, central lemma awns 13-18 $\mathrm{mm}$ long. Stipagrostia acutiflora

29b. Column twisted, 3-9 mm long, central lemma awns 25-60 $\mathrm{mm}$ long ... 30

30a. Glumes 3-veined, lower glumes glabrous .Stipagrostis plumosa

30b. Glumes 5-7-veined, lower glumes pilose. Stipagrostis multinerva

Group 6: Spikelets prickly; inflorescence a simple raceme or panicle

1a. Lower glumes well developed, modified into a long flat recurved tail, upper glumes usually smaller, enfolding the lemmas, tuberculate

Leptothrium senegalense

1b. Lower glumes very small or suppressed, upper glumes and lemmas about equal with raised veins bearing hooked bristles

2a. Apical spikelets sterile; upper glumes 7-veined Tragus racemosus

2b. All spikelets fertile; upper glumes 5 -veined Tragus berteronianus

\section{Group 7: Inflorescence tri- or dichotomously branched}

1a. Panicles trichotomously branched and rebranched; spikelets $2 \mathrm{~mm}$ long; pedicels terete, club-shaped, slender Sphenopus divaricatus 1b. Panicles dichotomously branched; spikelets 6-8 $\mathrm{mm}$ long; pedicels 3-angled.

2a. Spikelets 5-9 (-12)-flowered; glumes 3-5-veined Cutandia maritima

2b. Spikelets 2-4-flowered; glumes 1-veined .3

3a. Panicles partially enclosed in the sheath below; panicle internodes longer in length than the spikelets; lemmas 7.5- $8.5 \mathrm{~mm}$ long, apex awned, the awns 10-11 mm long . Cutandia memphetica

3b. Panicles exserted not enclosed in the sheath; panicle internodes shorter in length than the spikelets; lemmas 4.5-5.5 $\mathrm{mm}$ long, apex acute or bidentate

Cutandia dichotoma

\section{Group 8: Inflorescence subtended by spatheoles}

1a. Inflorescence a single terminal spikelet, one-sided enclosed by spatheole; lemmas villous.......Lygeum spartum

1b. Inflorescence composed of many spikelets; lemmas not villous

2a. Inflorescence composed of male and female racemes....... 3

2b. Inflorescence composed of bisexual florets ......................... 5

3a. Female racemes sessile subtended by bony utricles; male racemes pedunculate projecting from the utricles Coix lacryma-jobi

3b. Female racemes subtended by 1 or more herbaceous spatheoles

4a. Female inflorescences $2-5 \mathrm{~cm}$ broad; rachises thick woody, enclosed by several leafy spatheoles. Zea mays subsp. mays

4b. Female inflorescences less than $1 \mathrm{~cm}$ broad; rachises tough not woody, enclosed by single spatheole....

Zea mays subsp.mexicana

5a. Rachis with white hairs Hyparrhenia hirta

5b. Rachis pubescent or glabrous but not with white haris.... 6

6a. Spatheoles colored red or brown ...................................... 7

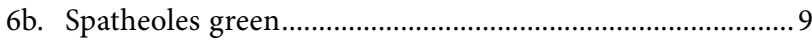

7a. Plants not aromatic; spikelets 6-14 $\mathrm{mm}$ long surrounded by hairs; callus bearded, the hairs red; lemma awns 20-70 $\mathrm{mm}$ long, straight, hispidulous Themeda triandra

7b. Plants aromatic; spikelets 4-5.5 mm long; callus not bearded; lemma awns 7-18 $\mathrm{mm}$ long, geniculate with a twisted column..... 8 
8a. Lower glume of sessile spikelet veins distinct, winged; lemma awns 12-18 $\mathrm{mm}$ long. Cymbopogon martini

8b. Lower glume of sessile spikelet veins absent or obscure and not winged; lemma awns 7-10 mm long...

Cymbopogon jwarancusa

9a. Rachis villous; spikelet pedicels villous; lemmas unawned. Cymbopogon citratus

9b. Rachis ciliate; spikelet pedicels ciliate; lemmas awned ... 10

10a. Awns geniculate with a twisted column .11

10b. Awns straight. 14

11a. Plants aromatic; racemes 2 12

11b. Plants not aromatic; racemes 1

12a. Lower glume of sessile spikelet elliptic lanceolate, usually 2 - or 3-veined between the keels ....... Cymbopogon nardus

12b. Lower glume of sessile spikelet narrowly lanceolate, usually veinless between the keels Cymbopogon flexuosus

13a. Lower glume of sessile spikelet pubescent with reddish hairs and not pitted, apex obtuse; lemma awns absent or to $10 \mathrm{~mm}$ long. Themeda villosa

13b. Lower glumes of sessile spikelet glabrous, shining, pitted, apex acute; lemma awns 12-18 $\mathrm{mm}$ long. Dichanthium foveolatum

14a. Racemes 2-3 cm long; spatheoles 2-3 cm long; lower glume of sessile spikelet glabrous .....

Cymbopogon schoenanthus subsp. schoenanthus

14b. Racemes 1-2 cm long; spatheoles 1-2 cm long; lower glume of sessile spikelet pubescent

.Cymbopogon schoenanthus subsp. proximus

Group 9: Inflorescence plumose, large 20-60 cm long, open; culms 4-8 $m$ tall

1a. Culms solid; callus bearded with white silky hairs. . .2

1b. Culms not solid; callus not bearded .3

2a. Callus hairs white; panicles $25-40 \mathrm{~cm}$ long Saccharum spontaneum

2b. Callus hairs off-white; panicles up to $1 \mathrm{~m}$ long ..Saccharum officinarum

3a. Lemmas pilose, the hairs 4-9 $\mathrm{mm}$ long; rachilla glabrous; glumes longer than the florets. Arundo donax

3b. Lemmas glabrous, rachilla hairy; glumes shorter than the florets 4a. Rachilla hairs 4-7 mm long; upper glumes 3-5(-6) mm long; leaf blades scabrid below..................Phragmites karka

4b. Rachilla hairs 8-15 mm long; upper glumes 6-9 mm long; leaf blades smooth below. Phragmites australis

Group 10: Inflorescence a simple spike, raceme or spikelike panicle

1a. Inflorescence a fragile cylindrical bilateral raceme with spikelets sunken in hollow of axes, glumes placed side by side covering the hollow

1b. Inflorescence not as above and without any sunken spikelets along the axis.

2a. Keel of glume wingless; culms and racemes strongly curved Parapholis incurva

2b. Keel of glume winged; culms and racemes usually straight

3a. Racemes bearing 5-10 spikelets; upper glume apices acute; lemma apices acute.

.Parapholis marginata

3b. Racemes bearing 10-20 spikelets; upper glume apices acuminate; lemmas apices obtuse..... Parapholis filiformis

4a. Spikes 1-sided . .5

4b. Spikes 2 - or more sided. 6

5a. Raceme not subtended by inflated leaf sheath; spikelets pectinate, solitary. Festuca pectinella

5b. Raceme subtended by an inflated leaf sheath; spikelets surrounding the rachis, not pectinately arranged. Tetrapogon cenchriformis

6a. Spikelets white-silky villous Lasiurus scindicus

6b. Spikelets green .7

7a. Raceme subtended by an inflated leaf sheath Elionurus royleanus

7b. Racemes not subtended by an inflated leaf sheath ............ 8

8a. Racemes partially enclosed in the sheath Dichanthium foveolatum

8b. Racemes exserted, not enclosed in the sheath .................... 9

9a. Inflorescence open, lax with remote spikelets. 10

9b. Inflorescence dense, narrow with approximate spikelets. 16

10a. Lower glumes absent; spikelets rotated $90^{\circ}$ edgewise from the culm axis and packed adaxially.

10b. Lower glumes present; spikelets not rotated $90^{\circ}$ edgewise from culm axis and packed laterally 
11a. Glumes much longer than the spikelets; lemmas ellipticovate, rigid Lolium temulentum

11b. Glumes shorter than the spikelets; lemmas oblong-lanceolate, not rigid..... ... 12

12a. Spikelets 11-22-flowered; lemmas awned, at least the upper ones ..Lolium multiflorum

12b. Spikelets 3-10-flowered; lemmas unawned, rarely awned ... 13

13a. Perennials; spikelets 2-10-flowered; lemma, if awned, up to $8 \mathrm{~mm}$ long; glumes $1 / 3$ to as long as the spikelets Lolium perenne

13b. Annuals; spikelets 5-8-flowered; lemma, if awned, the awns 3-10 $\mathrm{mm}$ long; glumes as long or longer than the spikelets.

Lolium rigidum

14a. Rachis fragile, disarticulating at the base of each internode, spikelets falling with attached rachis

Elymus farctus

14b. Rachis not disarticulating at maturity; florets falling separately, the glumes persistent 15

15a. Glumes unawned, apex obtuse or mucronate; lemmas unawned; rhizomes absent. Elymus elongatus

15b. Glumes mucronate or short-awned, apex acute; lemmas unawned or short-awned if awned, the awns up to $6 \mathrm{~mm}$ long; rhizomes present Elymus repens

16a. Inflorescence short, rarely over $10 \mathrm{~cm}$ long; internodes clavate; spikelets in pairs at each node of thick rachis, pedicelled spikelet sterile and sessile spikelet fertile, deeply sunken in excavated rachis Hemarthria altissima

16b. Inflorescence long, over $10 \mathrm{~cm}$; internodes not clavate; one to many spikelets borne at a node, not as above and not sunken in an excavated rachis.

17a. Glumes narrowly-linear throughout their length appearing awnlike.

17b. Glumes lanceolate to ovate, wider near the base and not appearing awnlike throughout. ... 19

18a. Inflorescence rachis glabrous throughout; lemmas 8-12 $\mathrm{mm}$ long, scabrous Taeniatherum caput-medusae

18b. Inflorescence rachis ciliate along the margins; lemmas 5-7 $\mathrm{mm}$ long, villous Crithopsis delileana

19a. Spikelets in pairs or in groups of 3 at a node .20

19b. Spikelets solitary at each node .26

20a. Spikelets in pairs, comprising 1 sessile and 1 pedicelled spikelet without rhachilla extension; glumes dissimilar; central lemma awned from a sinus; flexuous; 8-22 mm long. Pogonatherum paniceum 20b. Spikelets in group of 3 at each node, lateral spikelets staminate or sterile, central spikelets 1-flowered with bristlelike rachilla extension; glumes similar; central lemma awned from apex, not within a sinus 21

21a. Inflorescence rachis tough, not disarticulating at maturity. Hordeum vulgare

21b. Inflorescence rachis fragile, disarticulating at maturity......

22a. Awns of fertile lemma stout, 4-15 cm long; 2 lateral florets sessile; glumes silky hairy.......... Hordeum spontaneum

22b. Awns of fertile lemma slender, not more than $3 \mathrm{~cm}$ long; 2 lateral florets pedicelled; glumes not silky hairy ...........23

23a. Margin of glumes eciliate; lemma awns 10-24 mm long....

23b. Margin of glumes long ciliate; lemma awns $18^{-} 50 \mathrm{~mm}$ long... 25

24a. One glume of the lateral spikelets expanded below into a broad or narrow wing

Hordeum marinum subsp.marinum

24b. Both glumes of the lateral spikelets not expanded below into a broad or narrow wing.....

Hordeum marinum subsp. gussoneanum

25a. Leaf blades green; anthers of central spikelets $0.7-1.4 \mathrm{~mm}$ long; prolongation of rachilla of lateral spikelets $3-4 \mathrm{~mm}$ long, slender ...............Hordeum murinum subsp. leporinum

25b. Leaf blades glaucous; anthers of central spikelets $0.2-0.5$ $\mathrm{mm}$ long; prolongation of rachilla of lateral spikelets $1-2$ $\mathrm{mm}$, stout Hordeum murinum subsp. glaucum

26a. Glumes rounded over the midveins, lateral veins distinct.. 27

26b. Glumes keeled over the midveins, lateral veins not always distinct.

27a. Spikes moniliform (resembling a string of beads), swollen at base; disarticulating at the base of the spikelet Aegilops ventricosa

27b. Spikes not moniliform or swollen at base; disarticulating at the base of the internode. 28

28a. Spikes 10-20 cm long; glumes unawned; only the terminal spikelets with 2 long stout awns............ Aegilops longissima

28b. Spikes 1-8 cm long; glumes awned or unawned; terminal spikelet with awns no different than those below.

29a. Spikes 4-8 cm long; glume apex bidentate; principal lemma awns 30-60 $\mathrm{mm}$ long... Aegilops bicornis

29b. Spikes 0.5-5 cm long; glumes apex not bidentate; principal lemma awns $\leq 30 \mathrm{~mm}$ long 30 
30a. Glumes with veins unequal in width, unequally spaces and sunk into the surface of the glume

Aegilops geniculata

30b. Glumes with veins equal in width, equally spaced, riblike and protruding from the surface of the glume ....31

31a. Awns of lower glumes of equal width below and about equal in length; glumes of lowest fertile spikelet always with 3 awns. Aegilops kotschyi

31b. Awns of lower glumes of unequal width below and unequal in length; glumes of lowest fertile spikelet with 2 or 3 awns Aegilops peregrina

32a. Lemma awns up to $15 \mathrm{~cm}$ long (cultivated wheat) .33

32b. Lemma awns 0-7 mm long (wild plants) 36

33a. Inflorescense rachis fragile, disarticulating at maturity; upper glumes apex emarginate; lemma awns $10-15 \mathrm{~cm}$ long...... Triticum dicoccum

33b. Inflorescence rachis tough, not diararticulating at maturity; upper glumes apex with a unilateral teeth, truncate or 1-awned

34a. Glumes keeled above; winged on keeled; , rounded below; upper glumes apex with a unilateral teeth; truncate or 1-awned, awns 0-40 mm long; lemma awns 0-15 cm long Triticum aestivum

34b. Glumes keeled from the bases to the apex; upper glumes apex with unilateral teeth, truncate; lemma awns 8-15 $\mathrm{cm}$ long ...35

35a. Spikelets with 2-3 fertile florets. Triticum durum

35b. Spikelets with 3-5 fertile florets. Triticum turgidum

36a. Perennials; racemes tough; anthers 3-5 mm long Agropyron cristatum

36b. Annuals; racemes fragile; anthers $<1.5 \mathrm{~mm}$ long 37

37a. Palea at least as long as the body of the lemma; lemma awned, the awns 5-7 mm long; upper glumes awned, the awns 5-7 $\mathrm{mm}$ long Eremopyrum distans

37b. Palea shorter than the body of the lemma; lemma unawned or with a short awn, the awns up to $3 \mathrm{~mm}$ longupper glumes unawned Eremopyrum bonaepartis

\section{Group 11: Panicles spiciform, narrow usually $<1 \mathrm{~cm}$ wide}

1a. Panicles silky with the spikelets enveloped in long silky white hairs originating from the callus

Imperata cylindrica

1b. Panicles not silky and callus not long hairy..... . .2 2a. Base of glumes dilated forming a bulblike swelling .. Gastridium phleoides

2b. Base of glumes not dilated .3

3a. Panicle branches consisting of clusters each with 3 sterile spikelets \pm covering 2 smaller spikelets, one of which is fertile Lamarckia aurea

3b. Panicle branches not as above . .4

4a. Spikelets awned (lemma and/or glumes) .5

4b. Spikelets unawned. .13

5a. Glumes awned 6

5b. Glumes unawned; lemmas awned or mucronate ................9

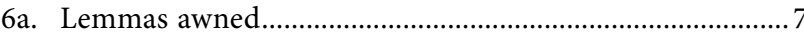

6b. Lemmas unawned .8

7a. Lower glume mucronate, the mucro 0.5-1 $\mathrm{mm}$ long; upper glume awns 1.5-6 mm long; fertile lemma apex entire; sterile lemma awn hooked. Holcus annuus

7b. Lower glume awns 3-7 mm long; upper glume awns 4-7 mm long; lemma apex dentate, 4-fid; lemma awns not hooked.

Polypogon monspeliensis

8a. Lower and upper glumes awns 1-2 mm long.....

Phleum pratense

8b. Lower and upper glumes awns 3-7 mm long Polypogon maritimus

9a. Lemma apex entire, mucronate or awned, the awns up to $2 \mathrm{~mm}$ long Trisetaria koeleroides

9b. Lemmas usually awned, the awns 1-16 mm long. 10

10a. Lemma awns 8-16 $\mathrm{mm}$ long, the geniculate awn arising dorsally near the base of the lemma.

Alopecurus myosuroides

10b. Lemma awns 1-5 mm long; the straight awn arising near or just below the apex of the lemma .. 11

11a. Glumes subequal, the lower minutely longer than the upper and often densely wooly Rostraria pumila

11b. Glumes unequal, the lower shorter and narrower than the upper, the lower never densely wooly, usually glabrous or with a few scattered hairs .12

12a. Panicles oval in outline, bristly; lemma surface setose, the hairs $0.5 \mathrm{~mm}$ long; lemma awns 3-5 mm long Rostraria hispida

12b. Panicles linear or oblong in outline, not bristly; lemma surface not setose; lemma awns 1-3 mm long 13 
13a. Panicles branches pubescent; lower glumes pubescent; lemma awns subterminal, scabrous............Rostraria rohlfsii

13b. Panicles branches glabrous; lower glumes glabrous or pubescent; lemma awns terminal, glabrous.

Rostraria cristata

14a. Glume keel winged .15

14b. Glume keel not winged .20

15a. Glume with 2 teethlike projections just above the middle.. Phalaris paradoxa

15b. Glume without 2 teethlike projections just above the middle 16

16a. Wings broad, margins erose or denticulate. .17

16b. Wings narrow, margins entire. .18

17a. Perennials; culms with a swollen base or pseudocorm; sterile spikelets surrounding the fertile spikelet; lemmas glabrous or with a few hairs near apex.

Phalaris coerulescens

17b. Annuals; culms without a swollen base; sterile spikelets not surrounding the fertile spikelet; lemmas pubescent.....

..Phalaris minor

18a. Inflorescence capitate, wider near base; caespitose annuals Phalaris canariensis

18b. Inflorescence oblong, not wider near base; rhizomatous perennials.

19a. Panicles interrupted below; rhizomes long and creeping; ligules 6-10 $\mathrm{mm}$ long Phalaris arundinacea

19b. Panicles not interrupted below; rhizomes short and knotty; ligules 2-4 mm long. Phalaris aquatica

20a. Lemmas pilose with tubercle-based hairs, the hairs 4-5 $\mathrm{mm}$ long... Melica persica

20b. Lemmas not long pilose or with tubercle based. .21

21a. Glumes deciduous. .22

21b. Glumes persistent .23

22a. Spikelets with 6-12 florets; palea keels pectinate-ciliate, the hairs nearly $1 \mathrm{~mm}$ long; ligule a ciliate membrane; lemma apex obtuse Eragrostis ciliaris

22b. Spikelets with 1 floret; palea keels not pectinate-ciliate; ligule a line of hairs; lemma apex acute

Sporobolus spicatus

23a. Lemma surface pubescent with clavate hairs; lemma apex truncate Phleum subulatum

23b. Lemma surface glabrous or pubescent but without clavate hairs; lemma apex acute or obtuse 24 24a. Culms 60-150 cm tall, erect and reedlike; spikelets $10-16$ $\mathrm{mm}$ long; panicles $7-22 \mathrm{~cm}$ long ....Calamagrostis arenaria

24b. Culms 5-50 cm tall, decumbent, geniculate or mat-forming; spikelets 2-7 mm long; panicles $1-7.5 \mathrm{~cm}$ long........25

25a. Spikelets 1-flowered, 2-2.5 mm long; lemmas 1-veined, 2-2.5 mm long; glumes shorter than the florets

Sporobolus alopecuroides

25b. Spikelets 3-5-flowered, 3-77 mm long; lemmas 5-veined, about $4 \mathrm{~mm}$ long; glumes slightly longer than the florets.. Rostraria obtusiflora

\section{Group 12: Panicles contracted, 0.5-3 (-4) cm wide}

1a. Upper and/or lower glumes awned 2

1b. All glumes unawned 3

2a. Lower glume awned, the awns 3-5 mm long; upper glume awns 7-8 $\mathrm{mm}$ long; lemmas 6-7 $\mathrm{mm}$ long; spikelets 7-10 $\mathrm{mm}$ long. Festuca brevis

2b. Lower glume unawned; upper glume awns 4-6 $\mathrm{mm}$ long; lemmas 8-18 mm long; spikelets $12-16 \mathrm{~mm}$ long. Festuca fasciculata

3a. Principal lemmas awned ... 4

3b. Principal lemmas unawned .. 9

4a. Lemmas hairy between the veins with 2-8 transverse rows of hair tufts; lemma awns 3-5 mm long. 5

4b. Lemmas not hairy between veins or with transverse rows of hair tufts; awns 5-15 mm long.

5a. Culms $50-120 \mathrm{~cm}$ tall, robust; panicles $17-35 \mathrm{~cm}$ long; anthers 1.6-2.7 $\mathrm{mm}$ long. Centropodia fragilis

5b. Culms $10-50 \mathrm{~cm}$ tall, smaller; panicles $2-15(-20) \mathrm{cm}$ long; anthers $0.7-1.3 \mathrm{~mm}$ long............Centropodia forskalii

6a. Lemma apex bidentate Bromus fasciculatus

6b. Lemmas apex entire. .7

7a. Panicles curved or nodding. Festuca myuros

7b. Panicles straight, not curved or nodding. 8

8a. Lemmas awns 5-12 mm long; callus rounded, about 0.2 mm long; glumes similar . Festuca bromoides

8b. Lemma awns 15-25 mm long; callus pointed, 0.5-0.8 mm long; glumes dissimilar Festuca fasciculata

9a. Spikelets 1-flowered 10

9b. Spikelets 3-10-flowered. 13 
10a. Lemmas 1-veined; ligule a line of hairs; glumes as long or shorter than the floret

10.b.Lemmas 3 or 5-veined; ligule membranous; glumes longer than the floret

11a. Leaf blades stiff and pungent, conspicuously distichous; glumes $1 / 2$ to as long as the floret; lower glume apex acute. Sporobolus pungens

11b. Leaf blades not stiff, pungent or distichous; glumes $<1 / 2$ as long as the floret; lower glume apex obtuse....

Sporobolus natalensis

12a. Glumes shiny and glabrous, scaberulous only along the keel; lemmas 1.5-2.3 mm long, apex obtuse; anthers 1-1.5 $\mathrm{mm}$ long Agrostis stolonifera

12b. Glumes scabrous throughout, roughened and not shiny; lemmas 1-1.5 mm long, apex truncate; anthers 0.5-0.8 $\mathrm{mm}$ long.

Polypogon viridis

13a. Lemmas 3-veined, 1.4-1.5 mm long, membranous, apex acute; glumes deciduous Eragrostis sarmentosa

13b. Lemmas 5-7-veined, 2-7.5 mm long, coriaceous, apex obtuse or truncate; glumes persistent. ... 14

14a. Spikelets with 3-10 fertile florets, if sterile florets present these located above the fertile ones; lemmas $2-2.5 \mathrm{~mm}$ long, apex obtuse; stamens 3 ; anthers about $0.3 \mathrm{~mm}$ long.

Catapodium rigidum

14b. Spikelets with a single upper fertile floret and two basal sterile florets; lemmas 3.5-7.5 mm long, apex truncate; stamens 6; anthers 3-4 $\mathrm{mml}$ long ............ Ehrharta calycina

\section{Group 13: Spikelets awned; panicles open}

1a. Lemma awns (8-)10-30 cm long, feathery .2

1b. Lemma awns much shorter, usually $<5 \mathrm{~cm}$ long, glabrous or scabrous

2a. Awns conspicuously twisted together with adjacent spikelet awns forming a tail; annuals Stipellula capensis

2b. Awns not conspicuously twisted together with others and not forming a tail; perennials

3a. Awns plumose for their whole length, the hairs $0.5-1.3$ $\mathrm{mm}$ long Stipa arabica

3b. Awns scabrid or pubescent, the hairs $<0.3 \mathrm{~mm}$ long ....... 4

4a. Awns 20-25 cm long, pubescent .................. Stipa lagascae

4b. Awns 6-13 cm long, scabrous................Stipellula parviflora

5a. Spikelets usually flushed with purple; awns of lemmas inserted from the base, the column with a clavate upper limb, brownish, with a ring of hairs at the junction Corynophorus divaricatus

5b. Spikelets green; awns of lemmas subapical or inserted near the middle, the column never clavate or with a ring of hairs at the junction 6

6a. Spikelets all solitary. 7

6b. Spikelets in pairs usually with a sessile and pedicellate or in triplets with two pedicellate and one sessile.................30

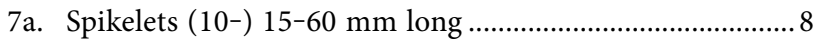

7b. Spikelets $1.5-12 \mathrm{~mm}$ long .36

8a. Glumes longer than the spikelet; lemma awns geniculate with a strongly twisted column; ovaries pubescent all over

8b. Glumes much shorter than the spikelet; lemma awns usually straight and not geniculate; ovaries pubescent only near the apex.

9a. Tips of lemmas bidentate with awned teeth, the teeth awns usually $1-1.5 \mathrm{~cm}$ long. 10

9b. Tips of lemmas bidentate with unawned teeth or with the awns of the teeth much shorter than $0.8 \mathrm{~mm}$ long .......... 12

10a. Spikelets erect; callus elongated, 4.2-6 $\mathrm{mm}$ long; lower glumes $25-40 \mathrm{~mm}$ long. Avena longiglumis

10b. Spikelets pendulous; callus obtuse, $<2 \mathrm{~mm}$ long; lower glumes 16-26 mm long.

11a. Spikelets (1.8-) 2-3 cm long; lower lemma 1.6-2 cm long. Avena barbata subsp. barbata

11b. Spikelets $1.4-1.8 \mathrm{~cm}$ long; lower lemma $1.2-1.4 \mathrm{~cm}$ long ... Avena barbata subsp. wiestii

12a. Floret callus glabrous; lemmas glabrous..........Avena sativa

12b. Floret callus hairy; lemmas hairy on the lower $1 / 3 \ldots \ldots . . .13$

13a. Lemmas dark brown; rachilla not disarticulating at maturity. Avena fatua

13b. Lemmas green; rachilla disarticulating between the florets at maturity 14

14a. Glumes 30-50 mm long; lowest lemmas 25-40 mm long.... Avena sterilis subsp. sterilis

14b. Glumes 25-30 mm long; lowest lemmas 20-25 mm long.... Avena sterilis subsp. ludoviciana

15a. Spikelets 45-60 mm long; lemmas awns 45-60 $\mathrm{mm}$ long.... 16

15b. Spikelets less than $45 \mathrm{~mm}$ long; lemmas awns 4-40 $\mathrm{mm}$ long 17 
16a. Panicles lax, spreading, broadly ovate, the branches longer than spikelets; base of lemmas in profile, contracted just above the callus; callus blunt with an oval scar..... Bromus diandrus var. diandrus

16b. Panicles contracted, stiffly erect, narrowly ovate, the branches usually shorter than the spikelets; base of lemmas in profile, continuous with callus; callus pointed with an elliptic scar Bromus diandrus var. rigidus

17a. Lower glumes 1-veined; upper glumes 3-veined; lemma awns not more than $20 \mathrm{~mm}$ long... ... 18

17b. Lower glumes 3-9-veined; upper glumes 5-13-veined; awns usually 15-40 $\mathrm{mm}$ long ...21

18a. Panicles branches compound bearing (3-) 5-8 fertile spikelets. Bromus tectorum var. tectorum

18b. Panicles branches simple bearing 1-3 fertile spikelets .... 19

19a. Panicles branches bearing 1-3 fertile spikelets; spikelet pedicels $\leq 1 \mathrm{~cm}$ long; lemmas 10-20 $\mathrm{mm}$ long... ...20

19b. Panicles branches bearing a single fertile spikelet; spikelet pedicels usually $>3 \mathrm{~cm}$ long; lemmas $15-40 \mathrm{~mm}$ long......... .. Bromus sterilis

20a. Plants perennial and rhizomatous; lemmas awnless, mucronate or short-awned, the awns up to $1.5 \mathrm{~mm}$ long.. Bromus inermis

20b. Plants annuals, caespitose; lemmas awned, the awns 12-20 $\mathrm{mm}$ long Bromus madritensis

21a. Lemma awns curved and reflexed-spreading. .22

21b. Lemma awns straight ......

22a. Lemmas coriaceous with inconspicuous veins; margins of lemma not involute but overlapping the back of the adjacent lemma; ligules 1-3 mm long. Bromus japonicus

22b. Lemmas membranous with conspicuous veins; margins of lemma somewhat involute at maturity, not overlapping the back of the adjacent lemma but wrapped around the caryopsis; ligules 3-6 mm long Bromus pulchellus

23a. Lemma awns briefly coiled at the base 24

23b. Lemmas awns not coiled at the base..... 26

24a. Spikelets 10-15 mm long, 2-3 mm wide; lemmas 6-11 $\mathrm{mm}$ long Bromus scoparius

24b. Spikelets (12-) 25-50 mm long, 3-16 mm wide, lemmas 11-18 $\mathrm{mm}$ long.

25a. Leaf blades 3-5 mm wide; lemma awns awns 11-20 mm long; spikelets 3-7 $\mathrm{mm}$ wide...... Bromus alopecuros

25b. Leaf blades 1-2.5 mm wide; lemma awns 6-12 $\mathrm{mm}$ long; spikelets 6-16 $\mathrm{mm}$ wide Bromus lanceolatus 26a. Lemmas strongly keeled; spikelets strongly laterally compressed. Bromus catharticus

26b. Lemmas rounded on the back; spikelets terete to moderately laterally compressed 27

27a. Glumes with ciliolate margins; culms nodes swollen Bromus aegyptiacus

27b. Glumes without ciliolate margins; culm nodes not swollen 28

28a. Lemmas 5.5-6.5 mm long; lemma awns 3-7 mm long, terete near base, straight; caryopsis longer than the palea, often visible beyond the tip of the lemma... Bromus lepidus

28b. Lemmas 8-17 mm long; lemma awns 5-17 mm long, flattened near base, straight or slightly divergent; caryopsis shorter than the palea, concealed within the floret.........29

29a. Lemmas 5-7-veined, 2-4 mm wide, glabrous or pubescent, narrowly lanceolate in profile; lemma awn arising 2-3 $\mathrm{mm}$ below the tip; anthers $1 \mathrm{~mm}$ long; lower glumes usually 3-veined

Bromus pectinatus

29b. Lemmas 7-9 (-11)-veined, 4.5-5.5 mm wide, hirsute, narrowly oblanceolate in profile; lemma awn arising 0.7-1.9 $\mathrm{mm}$ below the tip; anthers 2-2.5 mm long; lower glumes 3-7-veined. Bromus hordeaceus

30a. Spikelets in triplets with two pedicellate and one sessile 31

30b. Spikelets in pairs, fertile spikelets sessile and sterile spikelets pedicelled

31a. Panicles branches villous with white or dark brown hairs; fertile lemmas glabrous without transverse tufts of hairs; leaf blade apices not stiff and pungent, margins not white cartilaginous; upper glume of fertile lemma plumose .......... Chrysopogon plumulosus

31b. Panicle branches scaberulous without white or dark brown hairs; fertile lemmas with transverse tufts of hairs, the hairs 4-5 mm long; leaf blade apices stiff and pungent with conspicuous white cartilaginous margins; upper glume of fertile lemma unawned.....Danthoniopsis barbata

32a. Column of the lemma awns pubescent, hairy on the spiral; paleas present

32b. Column of the lemma awns glabrous, not hairy on the spiral; paleas absent or minute...... 34

33a. Apex of lower glume dentate, 3-fid; awns of upper lemma 10-16 mm long; plants perennial, rhizomatous Sorghum halepense

33b. Apex of lower glume entire; awns of upper lemma up to $10 \mathrm{~mm}$ long; plants annual or short-lived perennials, not rhizomatous ..... Sorghum bicolor

34a. Lower glumes pubescent with white or yellow hairs Sorghum arundinaceum 
34b. Lower glumes glabrous....... .35

35a. Panicle branches tough; spikelet callus pilose..... Sorghum $x$ drummondii

35b. Panicle branches fragile at node; spikelet callus not pilose. Sorghum virgatum

36a. Glumes and lemmas villous with white or purplish-red hairs

36b. Glumes and lemmas glabrous .37

37a. Spikelets 1.5-2 (-2.4) mm long; upper glumes not gibbous . Melinis minutiflora

37b. Spikelets 5-12 mm long; upper glumes gibbous.

Melinis repens

32a. Lemmas 1.5-2 mm long, the awns 3-5 mm long; apex of lemma obtuse Oloptum miliaceum

32b. Lemmas 6-7.5 mm long, the awns 8-13 mm long, apex of lemma acute Piptatherum holciforme

\section{Group 14: Spikelets unawned; panicles open}

1a. Both glumes absent or obscure .2

1b. Both glumes or at least the upper present..... .3

2a. Spikelets 8-11 mm long, 2.5-3.5 mm wide, persistent; lemma apex mucronate or awned, the awns up to 160 (cult.) Oryza sativa

2b. Spikelets 3.4-4.8 (-5.2) $\mathrm{mm}$ long, $1.2-1.4(-1.7) \mathrm{mm}$ wide, disarticulating entire; lemma apex entire, not mucronate or awned. Leersia hexandra

3a. Primary panicles branches whorled, at least on the lower nodes.

3b. Primary panicles branches not whorled at most nodes.. 10

4a. Lemmas pubescent, hairy below and along the veins ........5

4b. Lemmas glabrous throughout

5a. Lemma apex apiculate; anthers 1.4-2.6 mm long Poa diaphora

5b. Lemma apex not apiculate; anthers $0.3-0.7(-1) \mathrm{mm}$ long.. Poa persica

6a. Glumes longer than the florets, persistent. .7

6b. Glumes $\leq$ the florets, deciduous or persistent. .8

7a. Roots aromatic; lower glumes surface spinose, glumes dissimilar; spikelets $3.5^{-5} \mathrm{~mm}$ long.

Chrysopogon zizanioides 7b. Roots not aromatic; lower glumes surface not spinose, glumes similar; spikelets $1.8-3 \mathrm{~mm}$ long....

Agrostis stolonifera

8a. Spikelets dorsally compressed with a 5-veined sterile lemma just below the fertile lemma; leaf blades usually 12-35 mm wide Megathyrsus maximus

8b. Spikelets laterally compressed without a sterile lemma below the fertile lemma, the fertile lemma 1-veined; leaf blades $1-10 \mathrm{~mm}$ wide

\section{9}

9a. Panicles 3-20 cm long; apex of lower glumes obtuse; anthers $0.7-1 \mathrm{~mm}$ long. Sporobolus ioclados

9b. Panicles 20-60 cm long; apex of lower glumes acute; anther 1.1-1.3 $\mathrm{mm}$ long..... Sporobolus wrightii

10a. Spikelets pendulous; lemmas orbicular, gibbous, and auriculate near base .. 11

10b. Spikelets not pendulous; lemmas not orbicular. gibbous or auriculate near base....

11a. Spikelets 3-5 mm long, 3-5 mm wide; apex of lemma not cuspidate Briza minor

11b. Spikelets $14-25 \mathrm{~mm}$ long, 8-15 $\mathrm{mm}$ wide; apex of lemma cuspidate Briza maxima

12a. Spikelets in pairs with a fertile sessile and sterile pedicelled. Sorghum bicolor

12b. Spikelets not in pairs 13

13a. Lemmas with white capitate hairs near base, the hairs 0.2 $\mathrm{mm}$ long. Desmazeria philistaea

13b. Lemmas without white capitate hairs. 14

14a. Glumes and lemmas pilose with white tubercle-based hairs ..... Tricholaena teneriffae

14b. Glumes and lemmas not pilose or, if hairy, the hairs not white and tubercle-based.

15a. Lemma apex bifid and dentate ........................................ 16

15b. Lemmas apex not bifid or dentate.

16a. Spikelets 15-25 mm long; lemmas 10-13 mm long; ovary pubescent on the apex; rhizomatous perennials.

Bromus inermis

16b. Spikelets $\leq 7 \mathrm{~mm}$ long; lemmas 1.2-3.3 mm long; ovary glabrous throughout; caespitose annuals or perennials..17

17a. Spikelets 1.5-2.4 mm long, persistent; lower glumes without veins Melinis minutiflora

17b. Spikelets 4-7 mm long, deciduous with the pedicels; lower glumes 5-7-veined. 18

18a. Lemmas 2-3.5 mm long, apical lobes narrowly triangular, 
0.6-1.3 mm long; palea shorter than the lemma Schismus arabicus

18b. Lemmas $1.5-2 \mathrm{~mm}$ long, apical lobes broadly triangular, 0.3-0.4 mm long; palea $\geq$ the lemma in length

.Schismus barbatus

19a. Spikelets all 1-flowered with no additional florets Sporobolus wrightii

19b. Spikelets with more than a single floret .20

20a. Spikelets with one fertile floret and 1 or 2 sterile florets.....

20b. Spikelets with 3-10 fertile florets .28

21a. Spikelets laterally compressed, each spikelet with 2 basal sterile florets; fertile florets with 6 stamens

Ehrharta calycina

21b. Spikelets dorsally compressed, each spikelet with 1 basal sterile floret; fertile florets with 3 stamens.

22a. Upper fertile lemma faintly to strongly rugose. Megathyrsus maximus

22b. Upper fertile lemma smooth .23

23a. Spikelets 3.5-5.5 $\mathrm{mm}$ long 24

23b. Spikelets 2-3.2 (-3.5) $\mathrm{mm}$ long .25

24a. Plants annual without woody culms or resembling bushes; leaf blade apices not pungent Panicum miliaceum

24b. Plants perennial with woody culms resembling bushes; leaf blade apices pungent

Panicum turgidum

$25 \mathrm{a}$. Lower glumes $1 / 2-2 / 3$ the length of the spikelet; lemmas apex acute Panicum antidotale

$25 \mathrm{~b}$. Lower glumes up to $1 / 3$ the length of the spikelet...... 26

26a. Plants with a knotty rootstock; lower glume membranous, 1-3 (-7)-veined Panicum coloratum

26b. Plants without a knotty rootstock but with slender rhizomes or stolons; lower glumes hyaline, usually unveined, rarely 3 -veined.

27a. Plants erect with spreading rhizomes; leaf sheaths tough, wooly on the margins when young; leaf blade apices pungent; ligules $0.3-0.5 \mathrm{~mm}$ long. Panicum repens

27b. Plants with stolons; leaf sheaths loose, papery, glabrous on the margins; leaf blade apices not pungent; ligules $0.8-2$ mm long..... Panicum hygrocharis

28a. Lemmas 5-veined .29

28b. Lemmas 3-veined. 32

29a. Spikelets 10-18 mm long; lemmas 6-9 mm long; culms
50-150 cm tall; leaf blades 4-10 mm wide Lolium arundinaceum

29b. Spikelets 2-10 $\mathrm{mm}$ long; lemmas 2-5 mm long; culms $\leq$ $65 \mathrm{~cm}$ tall; leaf blades $1-5 \mathrm{~mm}$ wide 30

30a. Plants perennial forming a bulbous base composed of old leaf sheaths; anthers 1.5-2.5 mm long; apex of ligule acute ..Poa sinaica

30b. Plants annual or short-lived perennials, not forming a bulbous base of old leaf sheaths; anthers $\leq 1.1 \mathrm{~mm}$ long; apex of ligule obtuse.

. .31

31a. Anthers 0.6-1 (-1.1) mm long; spikelets crowded or sparsely arranged on the branches. Poa infirma

31b. Anthers 0.1-0.5 mm long; spikelets always crowded on the branches Poa annua

32a. Plants perennial, forming innovations at the basal nodes; caryopses narrowly triangular in cross section, strongly laterally flattened with a deep ventral groove Eragrostis tenuifolia

32b. Plants annual, usually tufted, without innovations at the basal nodes; caryopses not narrowly triangular in cross section or strongly laterally flattened, without a deep ventral groove

33a. Palea keels prominently ciliate, the cilia $0.2-1 \mathrm{~mm}$ long 34

33b. Palea keels smooth or scabrous the scabridities $<0.2 \mathrm{~mm}$ long.

34a. Spikelets 2.2-3 mm long; lemmas and culms without glands; lemmas (0.8-) 1-1.2 mm long, oblong; anthers $2 \ldots$

Eragrostis lepida

34b. Spikelets 6-20 mm long; lemmas with crateriform glands on the keels, similar glands also often present below the nodes; lemmas 2-2.8 mm long, broadly ovate; anthers 3 ... Eragrostis cilianensis

35a. Ligules membranous, neither ciliolate or ciliate

Eragrostis japonica

35b. Ligules membranous and ciliolate to ciliate, the cilia often longer than the basal membrane 36

36a. Plants with glandular pits or bands somewhere, the locations various, including any or all of the following: below the cauline nodes, on the sheaths, blades, rachises, panicle branches or pedicels, or on the keels of the lemmas and paleas 37

36b. Plants without glandular pits or bands 40

37a. Spikelets 1-1.4 mm wide; pedicels 1-10 mm long, lax appressed or divergent. ..Eragrostis pilosa

37b. Spikelets $1.1-4 \mathrm{~mm}$ wide; pedicels $0.2-4 \mathrm{~mm}$ long, stiff, straight, usually divergent

38 
38a. Lemmas 2-2.8 $\mathrm{mm}$ long with 1-3 crateriform glands along the keels; spikelets 6-20 $\mathrm{mm}$ long, 2-4 mm wide, with 10-40 florets; disarticulation below the florets, the rachillas persistent; anthers yellow ..... Eragrostis cilianensis

38a. Lemmas $1.4-1.8 \mathrm{~mm}$ long, rarely with 1 or 2 crateriform glands along the keels; spikelets 4-7 (-11) mm long, 1.1-2.2 mm wide, with 7-12 (-20) florets; disarticulation below the lemmas, both the paleas and rachillas usually persistent; anthers reddish-brown 39

39a. Panicles with glandular regions below the nodes, the glandular tissue forming a ring or band, often shiny or yellowish; blade margins without crateriform glands; pedicels without glandular bands .........Eragrostis barrelieri

39b. Panicles sometimes with glandular areas, but rarely rings of glandular spots or crateriform pits below the nodes, the glands usually dull greenish-gray to straminious; blade margins sometimes with crateriform glands; pedicels usually with glandular bands. Eragrostis minor

40a. Lemmas 1.6-3 mm long; florets persistent and the grain retained within; caryopses $0.3-1.3 \mathrm{~mm}$ long obovoid, smooth, light brown to white; plants cultivated, occasionally escaping.

Eragrostis tef

40b. Lemmas 1.2-1.7 mm long; florets deciduous, the grains not retained within; caryopses $0.4-0.9 \mathrm{~mm}$ long, ovoid, orbicular to prism-shaped or isodiametric, smooth or striate, brownish; plants native or established introductions.......

. .41

41a. Spikelets 5-25 mm long, 10-60-flowered; anthers 2 Eragrostis tremula

41b. Spikelets 3-15 mm long, 4-20 flowered; anthers 3 .42

42a. Lower glumes 1-1.5 mm long, about as long as the upper glume, 1-veined; panicle branches not whorled at the lower nodes; lemma apex truncate or obtuse; caryopses orbicular, isodiametric Eragrostis aspera

42b. Lower glumes $0.5-0.7 \mathrm{~mm}$ long, $1 / 2$ to $2 / 3$ as long as the upper glume, unveined; panicle branches whorled at the lower nodes; lemma apex acute or obtuse caryopses ellipsoid

43a. Panicle branches partially included below in the subtending leaf; spikelets 5-15 cm long; caryopses $0.5-0.7 \mathrm{~mm}$ long... Eragrostis aegyptiaca

43b. Panicle branches exserted and not included below in the subtending leaf; spikelets 3-6 mm long; caryopses 0.7-1 $\mathrm{mm}$ long. Eragrostis pilosa

\section{ACKNOWLEDGEMENTS}

We thank Alice Tangerini at the US National Herbarium for preparing Figures 1 and 2, Kitt Repass for preparing the illustrations, the Smithsonian Institution for permission to use all illustrations in Figure 2, and Kamal Ibrahim and two anonymous reviewers for suggesting improvements to the manuscript.

\section{REFERENCES}

Boulos L. 2009. Flora of Egypt checklist - Revised annotated edition. Cairo: Al Hadara Publishing.

Clayton W, Renvoize S. 1986. Genera graminum. Grasses of the world. Kew Bulletin, Additional Series 13: $1-389$.

Clayton WD, Vorontsova MS, Harman KT, Williamson H. 2016 onwards. GrassBase - The online World grass flora. The Board of Trustees, Royal Botanic Gardens http://www.kew.org/data/grasses-db.html (accessed 9 April 2019).

Cope TA. 2005. Gramineae. In: Boulos L (Ed) Flora of Egypt, vol 4: Monocotyledons (Alismataceae-Orchidaceae). Cairo: Al Hadara Publishing; p. 124-349.

Cope TA, Hosni A (1991) A key to Egyptian Grasses. Royal Botanic Gardens, Kew.

Herrera Arrieta YH, Peterson PM. 2018. Grasses of Chihuahua, Mexico. Smithsonian Contributions to Botany. 107: 1-380.

Hosni HA, Ibrahim KM. 2004. The identification of grasses of the northwestern coastal region of Egypt by their vegetative characters. Taeckholmia. 24: 79-89.

Ibrahim KM, Dube S, Peterson PM, Hosni HA. 2018. Grasses of Mali. Smithsonian Contributions to Botany. 108: 1-146.

Ibrahim KM, Hosnii HA, Peterson PM. 2016. Grasses of Egypt. Smithsonian Contributions to Botany. 103: $1-201$.

Kellogg EA (2015) Xlll. Flowering Plants, Monocots, Poaceae. In: Kubitski K (Ed) The Families and Genera of Vascular Plants. Switzerland: Springer International; p. 1-416.

Reveal JL. 2012. An outline of a classification scheme for extant flowering plants. Phytoneuron. 2012-37: 1-221.

Soreng RJ, Davidse G, Peterson PM, Zuloaga FO, Judziewicz EJ, Filgueiras TS, Morrone O. 2019. Catalogue of New World Grasses (Poaceae). http://wwwtropicosorg/project/cnwg (accessed 4 April 2019).

Soreng RJ, Peterson PM, Romaschenko K, Davidse G, Teisher JK, Clark LG, Barberá P, Gillespie LJ, Zuloaga FO. 2017. A worldwide phylogenetic classification of the Poaceae (Gramineae) II: An update and a comparison of two 2015 classifications. Journal of Systematics and Evolution. 55: 259-290. 
Soreng RJ, Peterson PM, Romaschenko K, Davidse G, Zuloaga FO, Judziewicz EJ, Filgueiras TS, Davis JI, Morrone O. 2015. A worldwide phylogenetic classification of the Poaceae (Gramineae). Journal of Systematics and Evolution. 53: 117-137.

Stevens PF. 2001 onwards. Angiosperm Phylogeny Website, Version 14, July 2017. http://wwwmobotorg/ MOBOT/research/APweb/ (accessed 4 April 2019).

Täckholm V. 1974. Students' Flora of Egypt, $2^{\text {nd }}$ edition. Cairo: Cairo University.

Täckholm V, Täckholm G, Drar M. 1941. Flora of Egypt vol. I. Pteridophyta, Gymnospermae and Angiospermae, Part Monocotyledones: Typhaceae- Gramineae. Bulletin of the Faculty of Science. 17: 1-557.

Takhtajan A. 2009. Flowering plants, ed. 2. Netherlands: Springer.

Tzvelev N. 1989. The system of grasses (Poaceae) and their evolution. The Botanical Review. 55: 141-204. 